\title{
Rigorous numerics for NLS: bound states, spectra, and controllability
}

\author{
Roberto Castelli*1 and Holger Teismann ${ }^{\dagger 2,1}$ \\ ${ }^{1}$ Basque Center for Applied Mathematics, Bilbao, Basque Country, Spain \\ ${ }^{2}$ Department of Mathematics and Statistics, Acadia University, Wolfville, Canada
}

October 24, 2013

\begin{abstract}
In this paper it is demonstrated how rigorous numerics may be applied to the one-dimensional nonlinear Schrödinger equation (NLS); specifically, to determining bound-state solutions and establishing certain spectral properties of the linearization. Since the results are rigorous, they can be used to complete a recent analytical proof [6] of the local exact controllability of NLS.
\end{abstract}

Key words: rigorous numerics, radii polynomials, controllability of PDEs, spectral analysis, BEC Subject classifications: 65G99, 35Q55, 35Q93

\section{Introduction}

Analytical proofs of interesting/important/desirable properties of mathematical models are often asymptotic in nature; as such, they are liable to leave a finite number of cases undecided. Or an analytical argument may reduce the property of interest to a criterion that needs to be verified each time the property is to be established for a particular model. In both cases, it is natural to resort to numerical methods to conclude the argument.If this reasoning is to have the stature of a rigorous proof, one needs to use rigorous numerics.

A famous case in point is the proof of the existence of the Lorentz attractor by Tucker [27, 28]; examples of this general scenario closer to the topic of the present paper include

- the asymptotic formula for the ground-state energy of a non-relativistic atom (by numerical verification of an elementary inequality) [16];

- the conditional asymptotic stability of solitary waves of the cubic nonlinear Schrödinger equation (by numerical verification of the gap condition) [14;

- the absence of imbedded eigenvalues for linearized NLS [20, 3];

- the existence of surface gap solitons of the 1-D NLS (by numerical verification of an integral inequality) [15];

- the exclosure of eigenvalues of the Schrödinger equation with a perturbed periodic potential 22 .

*rcastelli@bcamath.org

†hteisman@acadiau.ca 
In the present paper we apply this paradigm to spectral properties of the linearized NLS (on a finite interval with Dirichlet boundary conditions), which are needed in the analytical proof of its controllability [6].

Controlled manipulation of quantum systems is a very active field in science and engineering (several review papers and monographs are available on this subject; for a recent survey, see e.g. [7] and the literature therein). A class of systems that have been studied intensely are Bose Einstein condensates (BEC). In this paper we consider a one-dimensional condensate in a hard-wall trap ("condensate-ina-box"), where the trap size (box length) is a time-dependent function $L(t)$ that can be manipulated. The precise definition of the model is given in Section 2.1 below. Given an initial state, the objective is to "engineer" the control function $L(t)$ such that the condensate will be guided to a particular target state. The model we are considering was first proposed by Band et al. 4 to study adiabaticity in a nonlinear quantum system. More recently, the opposite regime, fast transitions ("shortcuts to adiabaticity"), has been investigated for BECs in box potentials [26, 13. Condensates in a box trap have actually been realized experimentally [21, an accomplishment that attracted considerable attention.

In light of the these developments, it is natural to study the mathematical control properties of the nonlinear Schrödinger equation. Ref. [6] establishes a local controllability result for (1a) in the vicinity of the nonlinear ground state $\phi$; the precise statement is given in Section 2.3 below.

The proof relies on two spectral properties of the operator $\mathcal{L}$ that arises by linearizing equation (4a) (the rescaled version of (1a)) about the state $\varphi(t, x)=e^{i \mu t} \phi(x)$; namely $\left(\Psi_{n}^{(1)}(x)\right.$ denotes the first component of the $n$-the eigenfunction of $\mathcal{L}^{*}$; see Section 2.4,

(A) the integrals $\Gamma_{n}=\int_{0}^{1}(x \phi)^{\prime}(x) \overline{\Psi_{n}^{(1)}(x)} d x$ are non-zero;

(B) the non-zero eigenvalues $\lambda_{n}$ of $\mathcal{L}$ are simple.

Analytical proofs of these properties are available, but the arguments are asymptotic in nature and are only applicable for (potentially) large eigenvalue indices $n$. So for a finite number of cases the validity of (A) and (B) is unclear (although it is proved that controllability holds generically; see Section 2.3 for the precise statement). By means of rigorous computation we close this gap; i.e. we give a rigorous computer-assisted proof that (A) and (B) hold for all $n$. Enclosure of the nonlinear ground state and the spectrum of $\mathcal{L}$ is accomplished by applying radii polynomials and suitable estimates for bi- and trilinear convolution terms arising from the nonlinearity.

This paper is organized as follows.

In Section 2.1 we state the original model and its rescaled version, which is the one we are working with throughout the paper. We discuss bound-state solutions (Section 2.2) and describe the control problem and result proved in [6] (Section 2.3). Section 2 closes with a description of the linearization of NLS (around a given bound state) whose spectral properties will be studied by means of rigorous numerics. In Section 3 we give a general outline of the radii-polynomial method for performing rigorous numerical computations. How this general method is applied to the NLS-problem at hand is described in Section 4 Specifically, we describe the rigorous determination of bound states (Section 4.1) and eigenvalues and eigenfunctions of the linearization (4.2). We also explain our method for verifying that the eigenvalues are simple (Section 4.3). The final two sections of the main body of the paper contain an overview of the numerical results (Section 5) and some concluding remarks (Section 6). There are two appendices at the end of the paper, which contain derivations of some the required estimates. 


\section{Nonlinear Schrödinger equation and control problem}

\subsection{Problem statement and rescaled equation}

Following Band et al. 4], we consider the "condensate-in-time-varying-box" problem

$$
\begin{aligned}
i \hbar \psi_{t} & =-\frac{\hbar^{2}}{2 m} \psi_{x x}-\sigma \kappa|\psi|^{2} \psi, \quad(x \in(0, L(t)), t \in(0, T)), \sigma= \pm 1 \\
\psi(t, 0) & =\psi(t, L(t))=0 \quad(t \in[0, T])
\end{aligned}
$$

where

(i) $\psi(t, x) \in \mathbb{C}$ is the wave function, which is assumed to be normalized; i.e.,

$$
\|\psi(t)\|_{\ell^{2}(0, L(t))}^{2}=\int_{0}^{L(t)}|\psi(t, x)|^{2} d x=1 .
$$

In the control problem, $\psi$ plays the role of the state.

(ii) $\hbar$ and $m$ are Planck's constant and the particle mass;

(iii) $\kappa>0$ is a nonlinearity parameter, derived from the scattering length and the particle number;

(iv) the signs of $\sigma$ correspond to the focussing $(\sigma=1)$ and de-focussing $(\sigma=-1)$ cases, respectively;

(v) $L_{0}>0$ is the initial (and final) length of the box (we will choose $L_{0}=1$ below);

(vi) $L:[0, T] \rightarrow(0, \infty)$ is a function such that $L(0)=L_{0}=L(T)$ and plays the role of the control.

Remark 1 i) This problem is a nonlinear variant of the control problem solved by K. Beauchard [5]. ii) The normalization condition (2) can formally be derived from (1a) \& (1b), since the density $\rho(t, x):=|\psi(t, x)|^{2}$ and the current $J(t, x):=\frac{\hbar}{m} \operatorname{Im}\left(\bar{\psi}(t, x) \psi_{x}(t, x)\right)$ satisfy the usual continuity equation $\rho_{t}=-J_{x}$.

To non-dimensionalize the problem and to transform it to the time-independent domain $(0,1)$, we introduce new variables [4],

$$
\psi(t, x):=\frac{\hbar}{\sqrt{2 \kappa m} L(t)} \varphi\left(\frac{\hbar}{2 m} \int_{0}^{t} \frac{d s}{L(s)^{2}} \frac{x}{L(t)}\right)=: \frac{\hbar}{\sqrt{2 \kappa m} L(t)} \varphi(\tau, \xi) .
$$

Moreover, defining $u(\tau)=\frac{2 m}{\hbar^{2}} L(t) \dot{L}(t)$ and renaming $\tau \rightarrow t, \xi \rightarrow x$, gives

$$
\begin{aligned}
i \varphi_{t} & =-\varphi_{x x}-\sigma|\varphi|^{2} \varphi-i u(t)(x \varphi)_{x}, \quad(x \in(0,1), t \in(0, T)) \\
\varphi(t, 0) & =\varphi(t, 1)=0 \quad(t \in[0, T]) .
\end{aligned}
$$

The spectral and control properties of $4 \mathrm{a})-(4 \mathrm{~b})$ are the subject of this paper.

\subsection{Bound states}

We are looking for stationary solutions to the problem $4 \mathrm{aa}$, $4 \mathrm{~b}$ ) (with $u(\tau) \equiv 0$ ). To this end, let

$$
\varphi(t, x)=e^{i \sigma \mu t} \phi(x)
$$

wher ${ }^{1} \phi=\phi(x)$ is a nonlinear bound state corresponding to the chemical potential $\mu$; i.e. a real solutions of the boundary value problem

$$
\begin{aligned}
-\phi^{\prime \prime} & +\sigma \mu \phi-\sigma \phi^{3}=0 \quad(x \in(0,1)) \\
\phi(0) & =\phi(1)=0 .
\end{aligned}
$$

\footnotetext{
${ }^{1}$ Note that the sign in the exponents of the time-dependent part of $\varphi$ depends on $\sigma$. In the de-focusing case, the definition of $\mu$ is the one favoured by physicists. The choice of sign in the focusing case is consistent with 24, which is one of our main references for analyzing the linearized equation.
} 
Explicit formulas for the solutions of $5 \mathrm{5a},(5 \mathrm{~b})$ are available in terms of Jacobian elliptic functions. If $j \in\{0,1,2, \ldots\}$, then $\phi_{j}^{ \pm}(x)$ will denote the (real-valued) solution of $5 \mathrm{a}, 5 \mathrm{~b}$, which possesses precisely $j$ zeros ("nodes") within the interval $(0,1)$. The node-less solution $\phi^{ \pm}:=\phi_{0}^{ \pm}$is referred to as the ground state; the solutions $\phi_{j}^{ \pm}(j \geq 1)$ with one or multiple nodes are called excited states. To find an explicit solution formula for $\phi_{j}^{+}$and $\phi_{j}^{-}$, we first solve the equation(s)

$$
\begin{aligned}
\text { focussing case }(\sigma=1) & \mu=4(j+1)^{2}\left(2 k^{2}-1\right) K(k)^{2}, \quad \mu \in\left[-\stackrel{\circ}{\mu}_{j}, \infty\right) \\
\text { de-focussing case }(\sigma=-1) & \mu=4(j+1)^{2}\left(k^{2}+1\right) K(k)^{2}, \quad \mu \in\left[\stackrel{\circ}{j}_{j}, \infty\right)
\end{aligned}
$$

for $k$, where $K(k)$ denotes the complete elliptic integral of the first kind (see, e.g. [1]) and $\stackrel{\leftrightarrow}{\mu}_{j}:=$ $(j+1)^{2} \pi^{2}$. Note that, since $K(k)$ is a strictly increasing continuous function of $k \in[0,1)$ satisfying $K(0)=\frac{\pi}{2}$ and $\lim _{k \rightarrow 1^{-}} K(k)=\infty$, equation 6a [resp. 6b] has exactly one solution $k=k_{j}^{+}(\mu)$ [resp. $\left.k=k_{j}^{-}(\mu)\right]$ for any choice of parameters $\mu \in\left[-\sigma \dot{\mu}_{j}, \infty\right)$ and $j \in\{0,1,2, \ldots\}$. Moreover, the functions $k_{j}^{ \pm}:\left[-\sigma \stackrel{\circ}{j}_{j}, \infty\right) \rightarrow[0,1)$ are continuous and strictly increasing as well, and satisfy $\lim _{s \rightarrow \infty} k_{j}^{ \pm}(s)=1$. Writing $k_{j}^{ \pm}=k_{j}^{ \pm}(\mu)$, the solutions $\phi_{j}^{ \pm}$of $[5 \mathrm{a}, 5 \mathrm{~b}$ are given by [8], [9]

$$
\begin{aligned}
\phi_{j}^{+}(x) & =2 \sqrt{2}(j+1) k_{j}^{+} K\left(k_{j}^{+}\right) \operatorname{cn}\left(2(j+1) K\left(k_{j}^{+}\right)\left(x-\frac{1}{2}\right)+[j]_{2} K\left(k_{j}^{+}\right), k_{j}^{+}\right), \\
\phi_{j}^{-}(x) & =2 \sqrt{2}(j+1) k_{j}^{-} K\left(k_{j}^{-}\right) \operatorname{sn}\left(2(j+1) K\left(k_{j}^{-}\right) x, k_{j}^{-}\right)
\end{aligned}
$$

where $\mathrm{cn}=\mathrm{cn}(x, k)$ and $\mathrm{sn}=\mathrm{sn}(x, k)$ are the Jacobian elliptic cosine and sine functions, respectively, and $[j]_{2}:=j \bmod 2$.

\subsection{Control problem}

We now state the controllability result mentioned above, which roughly states that, "generically" w.r.t. the parameter $\mu$, exact controllability holds locally around the ground state. Here "generic" means the existence of an at most countable set $J \subset\left(-\sigma \pi^{2}, \infty\right)$ of potentially exceptional $\mu$ values. Defining

$$
\mathcal{H}:=\left\{f \in H^{3}(0,1 ; \mathbb{C}) \mid f(0)=f(1)=0\right\} \quad \text { and } \quad \mathcal{S}:=\mathcal{H} \cap\left\{\left.f \in L^{2}(0,1 ; \mathbb{C})\left|\int_{0}^{1}\right| f\right|^{2} d x=1\right\},
$$

the precise statement reads

Theorem 1 [6] Let $\mu \in\left(-\sigma \pi^{2}, \infty\right) \backslash J, \phi=\phi_{\mu}$ the corresponding ground state, and $T>0$. Then there exists a number $\delta=\delta(T, \mu)>0$ such that for all states $\varphi_{0}, \varphi_{1} \in \mathcal{S}$ satisfying

$$
\left\|\varphi_{0}-\phi\right\|<\delta \quad \text { and } \quad\left\|\varphi_{1}-e^{i \sigma \mu T} \phi\right\|<\delta
$$

there exists a control function $u \in H^{1}([0, T], \mathbb{R})$ with $\int_{0}^{T} u(t) d t=0$ such that the unique solution $\varphi \in C([0, T], \mathcal{H})$ of $4 a]$ - $4 b$ satisfies $\varphi(0)=\varphi_{0}$ and $\varphi(T)=\varphi_{1}$.

The fact that the theorem cannot be stated for all values of $\mu$ is due to the asymptotic nature of the direct analytical proof of the properties (A) and (B), which only covers (potentially) large $n$. Properties (A) and (B) can still be established generically (i.e. up to an at most countable set $J$ of possible exceptions) in an indirect way by using the analytic dependence of the operator $\mathcal{L}$ and its spectrum on the parameter $\mu$. However, while the genericity property implies that controllability holds with "probability one w.r.t. random choices" of $\mu$, for any particular value of $\mu$ the theorem can only be applied if (A) and (B) are verified for the finite number of cases not covered by the direct proof. In the remainder of the paper we are going to demonstrate that this verification can be accomplished by rigorous numerical computation. 


\subsection{Linearization}

The proof of Theorem 11 uses linearization around the ground state and the Implicit Function Theorem. If $\phi$ is a bound state, then the function $\varphi(t, x)=e^{i \sigma \mu t} \phi(x)$ is the unique solution of (4a)- $4 \mathrm{~b}$ ) with $\varphi(0, x)=\phi(x)$. Now we linearize around $\varphi$. The result is

$$
\begin{aligned}
i z_{t} & =-z_{x x}-\sigma|\varphi|^{2} z-2 \sigma \operatorname{Re}(\varphi \bar{z}) \varphi \\
z(t, 0) & =z(t, 1)=0 .
\end{aligned}
$$

The time dependence of the term involving $\operatorname{Re}(\ldots)$ is eliminated by the transformation $e^{i \sigma \mu t} \tilde{z}(t):=$ $z(t)$, which yields the BVP

$$
\begin{aligned}
i \tilde{z}_{t} & =-\tilde{z}_{x x}+\sigma \mu \tilde{z}-\sigma \phi^{2} \tilde{z}-2 \sigma \phi^{2} \operatorname{Re}(\tilde{z}) \\
\tilde{z}(t, 0) & =\tilde{z}(t, 1)=0
\end{aligned}
$$

It is natural to work with the real $(2 \times 2)$-system arising from $9 \mathrm{a}-(9 \mathrm{~b})$ by decomposition in real and imaginary parts. Consider the matrix operator

$$
\mathcal{L}:=\left(\begin{array}{cc}
0 & -\Delta+\sigma \mu-\sigma \phi^{2}(x) \\
\Delta-\sigma \mu+3 \sigma \phi^{2}(x) & 0
\end{array}\right)=:\left(\begin{array}{cc}
0 & L_{-} \\
-L_{+} & 0
\end{array}\right)
$$

( $\Delta$ denotes the one-dimensional "Laplacian" $\frac{d^{2}}{d x^{2}}$.) Then eq. 9a takes the form

$$
Z_{t}=\mathcal{L} Z
$$

where $Z(t, x)=\left(\begin{array}{l}\operatorname{Re}(\tilde{z}(t, x)) \\ \operatorname{Im}(\tilde{z}(t, x))\end{array}\right)$. The operator $\mathcal{L}$ is the main object of study.

\subsubsection{Provable properties of the spectrum of $\mathcal{L}$ (and $\mathcal{L}^{*}$ ) if $\phi$ is the ground state}

(i) The spectrum of $\mathcal{L}$ consists of eigenvalues only

(ii) all non-zero eigenvalues $\left\{\lambda_{n}, \bar{\lambda}_{n}\right\}_{n \geq 1}$ are purely imaginary, i.e.

$$
\lambda_{n}=i \beta_{n}, \quad \bar{\lambda}_{n}=-i \beta_{n}, \quad \beta_{n}>0 \quad(\forall n \geq 1) .
$$

(iii) the multiplicity of the eigenvalues is at most 2;

(iv) all, but possibly finitely many, non-zero eigenvalues are simple

(v) the multiplicity of the eigenvalue zero is 2 ; let

$$
\Phi_{0}^{+}=\left(\begin{array}{l}
0 \\
\phi
\end{array}\right) \quad \text { and } \quad \Phi_{0}^{-}=\left(\begin{array}{c}
\partial_{\mu} \phi \\
0
\end{array}\right) .
$$

Then

$$
\mathcal{L} \Phi_{0}^{-}=\Phi_{0}^{+} \quad \text { and } \quad \mathcal{L} \Phi_{0}^{+}=0 .
$$

and the vectors $\Phi_{0}^{+}, \Phi_{0}^{-}$form a basis of the generalized null space for $\mathcal{L}$.

(vi) Notation: $\Phi_{1}^{+}, \Phi_{2}^{+}, \ldots, \Phi_{1}^{-}, \Phi_{2}^{-}, \ldots$ denote the eigenvectors $2^{2}$ corresponding to the non-zero eigenvalues $\lambda_{1}, \lambda_{2}, \ldots, \bar{\lambda}_{1}, \bar{\lambda}_{2}, \ldots ;$ i.e.,

$$
\mathcal{L} \Phi_{n}^{ \pm}= \pm i \beta_{n} \Phi_{n}^{ \pm}, \quad \beta_{n}>0 \quad(n \geq 1)
$$

and $\overline{\Phi_{n}^{+}}=\Phi_{n}^{-}$for all $n \geq 1$ (where $\overline{(.)}$ denotes complex conjugation).

\footnotetext{
${ }^{2}$ Clearly, these are unique up to normalization.
} 
(vii) Similarly, $\Psi_{0}^{+}, \Psi_{0}^{-}, \Psi_{1}^{+}, \Psi_{2}^{+}, \ldots, \Psi_{1}^{-}, \Psi_{2}^{-}, \ldots$ denote the eigenfunctions for $\mathcal{L}^{*}$ with corresponding eigenvalues $\bar{\lambda}_{n}$ and $\lambda_{n}$, respectively; i.e.,

$$
\mathcal{L}^{*} \Psi_{n}^{ \pm}=\mp i \beta_{n} \Psi_{n}^{ \pm}, \quad \beta_{n}>0 \quad(n \geq 1)
$$

Moreover,

$$
\mathcal{L}^{*} \Psi_{0}^{+}=\Psi_{0}^{-} \quad \text { and } \quad \mathcal{L}^{*} \Psi_{0}^{-}=0 .
$$

where

$$
\begin{aligned}
& \Psi_{0}^{-}=\left(\begin{array}{c}
\phi \\
0
\end{array}\right) \\
& \Psi_{0}^{+}=\left(\begin{array}{c}
0 \\
\partial_{\mu} \phi
\end{array}\right) \\
& \overline{\Psi_{n}^{+}}=\Psi_{n}^{-}
\end{aligned}
$$

(viii) $\left\{\Phi_{m}^{ \pm}\right\}_{m \geq 0},\left\{\Psi_{n}^{ \pm}\right\}_{n \geq 0}$ form bi-orthogonal systems; i.e.,

$$
\left\langle\Phi_{m}^{\sigma}, \Psi_{n}^{\tau}\right\rangle=\delta_{m, n}^{\sigma, \tau}, \quad m, n \in\{0,1,2, \ldots\}, \sigma, \tau \in\{+,-\}
$$

where the inner product $\langle.,$.$\rangle is defined by$

$$
\langle U, V\rangle=\left\langle\left(\begin{array}{c}
U^{(1)} \\
U^{(2)}
\end{array}\right),\left(\begin{array}{c}
V^{(1)} \\
V^{(2)}
\end{array}\right)\right\rangle=\int_{0}^{1} U^{(1)}(x) \overline{V^{(1)}(x)} d x+\int_{0}^{1} U^{(2)}(x) \overline{V^{(2)}(x)} d x .
$$

and

$$
\delta_{m, n}^{\sigma, \tau}=\left\{\begin{array}{ll}
1, & m=n \text { and } \sigma=\tau \\
0, & \text { otherwise }
\end{array} .\right.
$$

Remark 2 Note that (other than in Section 2.2 above) the \pm superscripts do not refer to the focussing and defocusing cases here. Note also that the eigenfunctions $\Phi_{1}^{+}, \Phi_{2}^{+}, \ldots, \Phi_{1}^{-}, \Phi_{2}^{-}, \ldots$ and $\Psi_{1}^{+}, \Psi_{2}^{+}, \ldots, \Psi_{1}^{-}, \Psi_{2}^{-}, \ldots$ are complex-valued.

\subsubsection{A change of variables}

It is convenient to employ a similarity transformation [24, (12.15)]: Let

$$
J:=\left(\begin{array}{rr}
1 & i \\
1 & -i
\end{array}\right)
$$

Then

$$
\begin{aligned}
& i \mathcal{L}=J^{-1} \mathcal{M} J, \quad-i \mathcal{L}^{*}=J^{-1} \mathcal{N} J \quad \text { and so } \\
& \operatorname{spec}(\mathcal{L})=i \operatorname{spec}(\mathcal{M}), \quad \operatorname{spec}\left(\mathcal{L}^{*}\right)=-i \operatorname{spec}(\mathcal{N}),
\end{aligned}
$$

where

$$
\begin{aligned}
\mathcal{M} & :=\left(\begin{array}{rr}
-\Delta & 0 \\
0 & \Delta
\end{array}\right)+\sigma\left(\begin{array}{cc}
\mu-2 \phi^{2} & -\phi^{2} \\
\phi^{2} & -\mu+2 \phi^{2}
\end{array}\right) \\
\mathcal{N} & :=\left(\begin{array}{rc}
-\Delta & 0 \\
0 & \Delta
\end{array}\right)+\sigma\left(\begin{array}{cc}
\mu-2 \phi^{2} & \phi^{2} \\
-\phi^{2} & -\mu+2 \phi^{2}
\end{array}\right) .
\end{aligned}
$$

Now let $\left( \pm \beta_{n}, V_{n}^{ \pm}\right)$and $\left( \pm \beta_{n}, W_{n}^{ \pm}\right), \beta_{n} \geq 0$, be the eigenpairs for the operators $\mathcal{M}$ and $\mathcal{N}$, respectively, i.e., for $n \geq 1, \beta_{n}>0$,

$$
\begin{array}{rlr}
\Phi_{n}^{ \pm} & =J^{-1} V_{n}^{\mp}, \quad \Psi_{n}^{ \pm}=J^{-1} W_{n}^{\mp}, \\
\mathcal{M} V_{n}^{ \pm} & = \pm \beta_{n} V_{n}^{ \pm}, \quad \mathcal{N} W_{n}^{ \pm}= \pm \beta_{n} W_{n}^{ \pm} .
\end{array}
$$


Writing $\beta= \pm \beta_{n}, V=V_{n}^{ \pm}=\left(\begin{array}{l}u \\ v\end{array}\right), W=W_{n}^{ \pm}=\left(\begin{array}{l}w \\ z\end{array}\right)$, the characteristic equations $18 \mathrm{~b}$ are equivalent to the BVP

$$
\begin{aligned}
u^{\prime \prime}-(\sigma \mu-\beta) u & =-\sigma \phi^{2}(2 u+v), & u(0)=u(1)=0 \\
v^{\prime \prime}-(\sigma \mu+\beta) v & =-\sigma \phi^{2}(u+2 v), & v(0)=v(1)=0 \\
w^{\prime \prime}-(\sigma \mu-\beta) w & =-\sigma \phi^{2}(2 w-z), & w(0)=w(1)=0 \\
z^{\prime \prime}-(\sigma \mu+\beta) z & =-\sigma \phi^{2}(-w+2 z), & z(0)=z(1)=0 .
\end{aligned}
$$

\section{Computational method: rigorous computation using radii polynomials}

In this section we describe the rigorous computational method that will be used to

(P1) enclose the function $\phi(x)$ solution of $(5 \mathrm{a}),(5 \mathrm{~b})$;

(P2) enclose the eigenpairs $(\beta, W)$ solutions of $19 \mathrm{c}, 19 \mathrm{~d})$;

(P3) prove that the eigenvalues $\beta$ are simple.

These computations are based on suitable adaptations of the general method known as radii polynomials. The radii-polynomial approach, first introduced in [12], aims at demonstrating existence and local uniqueness of solutions of nonlinear problems by verifying the hypothesis of the contraction mapping theorem in Banach spaces. In recent years this technique has been successfully applied to a variety of nonlinear problems; see e.g 29, 30, 10, 11, and the references therein.

Before outlining the main steps of the method, as applied to P1, P2, and P3 above, we introduce some notation. For $z \in \mathbb{C}$, denote by $|z|=\max \{|\operatorname{Re}(z)|,|\operatorname{Im}(z)|\}$ and for a matrix $V=\left\{V_{i, j}\right\} \in \mathbb{C}^{n \times m}$ denote by $|V|=\left\{\left|V_{i, j}\right|\right\}$ and $|V|_{\infty}=\max _{i, j}\left|V_{i, j}\right|$. Given two matrices $A, B \in \mathbb{R}^{n \times m}$ the inequality $A<B$ is to be interpreted componentwise, i.e. $A_{i, j}<B_{i, j}$, for all $i, j$. Define the weights $w_{k}$ as

$$
w_{k}=\left\{\begin{array}{ll}
1, & k=0 \\
|k|, & k \neq 0
\end{array} .\right.
$$

If $x=\left\{x_{k}\right\}_{k \geq 0} \in\left(\mathbb{K}^{d}\right)^{\mathbb{N}}$ is a sequence in $\mathbb{K}^{d}$ (for some $d \geq 1$ and $\mathbb{K}=\mathbb{C}$ or $\mathbb{K}=\mathbb{R}$ ) and $s>0$, we define the $s$-norm of $x$ as

$$
\|x\|_{s}=\sup _{k}\left\{\left|x_{k}\right|_{\infty} w_{k}^{s}\right\}
$$

and $X^{s}$ the space

$$
X^{s}=\left\{x \in\left(\mathbb{K}^{d}\right)^{\mathbb{N}} \mid\|x\|_{s}<\infty\right\} .
$$

The space $\left(X^{s},\|\cdot\|_{s}\right)$ is a Banach space; we refer to $s$ as the decay rate parameter. Let $B(r)=\{x \in$ $\left.X^{s} \mid\|x\|_{s} \leq r\right\}$ be the ball of radius $r$ in $X^{s}$ and, for any $x \in X^{s}$, denote by

$$
B_{x}(r)=x+B(r)
$$

the ball centred at $x$.

The first step of the method consists of rephrasing the original problem in terms of an equation of the form

$$
f(x)=0,
$$

where $f: X \rightarrow W$ is a (possibly) nonlinear operator with $X=X^{s_{1}}, W=X^{s_{2}}$ and suitable $s_{1}, s_{2}$. Then we choose the finite dimensional parameter $m \geq 1$ and define the finite dimensional projections $\Pi_{m}^{X}: X \rightarrow X_{m}$ and $\Pi_{m}^{W}: W \rightarrow W_{m}$ as well as the infinite "tail projections" $\Pi_{\infty}^{X}: X \rightarrow X_{\infty}$ and $\Pi_{\infty}^{W}: W \rightarrow W_{\infty}$ by

$$
\Pi_{m}^{X}(x)=x^{(m)}=\left(x_{0}, \ldots, x_{m}\right), \quad \Pi_{\infty}^{X}(x)=x^{\infty}=\left(x_{m+1}, x_{m+2}, \ldots\right),
$$


and similarly for $\Pi_{m}^{W}$ and $\Pi_{\infty}^{W}$.

Consider the finite dimensional projection of the map $f$,

$$
\begin{aligned}
f^{(m)}: & X_{m} \rightarrow W_{m} \\
& x \mapsto f^{(m)}(x):=\Pi_{m}^{W} f\left(x, 0^{\infty}\right),
\end{aligned}
$$

and suppose that an approximate solution $\bar{x} \in X^{(m)}$ of $f^{(m)}(x)=0$ has been computed. Sightly abusing notation, we use $\bar{x}$ to indicate both the vector in $X^{(m)}$ and the sequence $\left(\bar{x}, 0^{\infty}\right) \in X$. Hence we also refer to $\bar{x}$ as the approximate zero of the full (infinite dimensional) map $f$, i.e.

$$
f(\bar{x}) \approx 0 .
$$

The next step is to define a nonlinear operator $T: X \rightarrow X$ with the property that the zeros of $f(x)$ are in one-to-one correspondence with the fixed points of $T$. The fixed point operator $T$ will be defined as a modified Newton operator centred at the numerical solution $\bar{x}$. The crux of the method is to prove that $T$ is a contraction.

Let

$$
D f^{(m)}:=\frac{\partial f^{(m)}}{\partial x^{(m)}}(\bar{x})
$$

be the Jacobian of $f^{(m)}$ evaluated at $\bar{x}$,

$$
\Lambda_{k}=\frac{\partial f_{k}}{\partial x_{k}}(\bar{x}) \quad(k=0,1,2, \ldots),
$$

and $A^{(m)} \in \mathbb{K}^{(m+1) \times(m+1)}$ an invertible approximate inverse of $D f^{(m)}$. Then we define the operator $A$ by

$$
(A x)_{k}:= \begin{cases}\left(A^{(m)} x^{(m)}\right)_{k}, & k \leq m \\ \Lambda_{k}^{-1} x_{k}, & k>m\end{cases}
$$

and the fixed point operator $T: X \rightarrow X$ as

$$
T(x)=x-A f(x) .
$$

To ensure that fixed points for $T$ correspond to zeros of $f(x)$, we need to prove that the operator $A$ is injective. Since the finite part $A^{(m)}$ is invertible by construction, this amounts to verifying that for $k>m$ the operators $\Lambda_{k}$ are invertible as well.

The existence (and uniqueness) of the fixed point for $T$ will follow from Banach's fixed point theorem, once the operator $T$ has been proven to be a contraction on a suitable subset of $X$. The candidate sets are the balls $B_{\bar{x}}(r)$ defined in $(20)$, hence we need rigorous estimates for the image of $T$ and the rate of contractivity of $T$ on these balls.

Suppose that, for a fixed computational parameter $M$, we have found bounds $Y=\left\{Y_{k}\right\}_{k<M}, Z=$ $\left\{Z_{k}(r)\right\}_{k<M}, Y_{M}$, and $Z_{M}(r)$, such that

$$
\left|(T(\bar{x})-\bar{x})_{k}\right| \leq Y_{k}, \quad \sup _{b_{1}, b_{2} \in B(r)}\left|\left[D T\left(\bar{x}+b_{1}\right) b_{2}\right]_{k}\right| \leq Z_{k}(r) \quad(\forall k<M),
$$

and

$$
\left|(T(\bar{x})-\bar{x})_{k}\right|_{\infty} \leq \frac{1}{w_{k}^{s}} Y_{M}, \quad \sup _{b_{1}, b_{2} \in B(r)}\left|\left[D T\left(\bar{x}+b_{1}\right) b_{2}\right]_{k}\right|_{\infty} \leq \frac{1}{w_{k}^{s}} Z_{M}(r) \quad(\forall k \geq M) .
$$

Definition 1 The radii polynomials are defined as

$$
\begin{aligned}
& p_{k}(r):=\left|Y_{k}+Z_{k}(r)\right|_{\infty}-\frac{r}{w_{k} s}, \quad k=0, \ldots, M-1, \\
& p_{M}(r):=Y_{M}+Z_{M}(r)-r .
\end{aligned}
$$


These are called "polynomials" because each bound $Z_{k}$ will be constructed as a polynomial in the variable $r$ with degree equal to the degree of nonlinearity of the map $f(x)$.

The final step in the procedure is to solve the inequalities $p_{k}(r)<0$ for $r$. Then $T$ will be a contraction in any ball $B_{\bar{x}}\left(r^{*}\right)$ whose radius $r^{*}$ satisfies $p_{k}\left(r^{*}\right)<0$ for all $k \in\{0,1, \ldots M\}$. This is the content of the next theorem.

Theorem 2 Suppose $Y=\left\{Y_{k}\right\}_{k}, Z(r)=\left\{Z_{k}(r)\right\}_{k}$ satisfy (24) for $k=1, \ldots, M-1$ and $Y_{M}, Z_{M}$ satisfy 25) and let the polynomials $p_{k}(r), p_{M}(r)$ be defined by (26). Then, for every number $r>0$ such that $p_{k}(r)<0$ for all $k=0, \ldots, M$, there exists a unique $x^{*} \in B_{\bar{x}}(r)$ such that $f\left(x^{*}\right)=0$.

Proof. See [12].

The enclosure radius $r$ arises as a solution of $p_{k}(r)<0(k=0, \ldots, M)$, where the polynomials $p_{k}(r)$ are constructed from analytical estimates and numerical computations. Although the method relies on computer calculations, the results are mathematically rigorous because all computations are performed in interval arithmetic (using the software package INTLAB [25]), which accounts for all possible rounding errors.

In addition to the radii-polynomial technique, there are several other computational methods based on the Contraction Mapping Principle (CMP), such as the Krawczyk operator approach [19, 17] or the methods developed by Yamamoto [31, by Koch et al. [2], and by Nagatou et al. 23]. The main difference is that in the the radii-polynomials approach the enclosure radius $r$ is computed a posteriori and optimally, whereas in most of the other methods an initial guess is made of the set on which $T$ might be contractive, and the hypotheses of the CMP are verified after the fact. We feel that our approach has at least two advantages: the use of interval arithmetic is deferred to the end of the process reducing computing time, and the procedure attempts to determine an enclosure radius that is as small as possible. The second consideration is particularly relevant to this work: the computation of the spectrum of $\mathcal{L}$ requires prior computation of $\phi(x)$ and the size of the intervals can growth dramatically when a large number of interval computations is performed; it is therefore necessary to have a very narrow enclosure of the solution $\phi(x)$. To accomplish this, we need sharp analytical estimates to control the truncation error arising from the finite dimensional approximation.

In summary, the technique consists of the following steps:

1. to formulate the problem in the form $f(x)=0$ for a suitable map $f: X \rightarrow W$;

2. to fix a finite-dimensional projection, compute a numerical solution $\bar{x}$, and construct the fixed point operator $T$;

3. to compute the bounds $Y_{k}, Z_{k}, Y_{M}$ and $Z_{M}$ and construct the radii-polynomials;

4. to determine $r$ such that $p_{k}(r)<0$.

\subsection{Construction of the radii polynomials}

The construction of the bounds $Y$ and $Z$ is described next. First we fix a computational parameter $M,(M>m)$, and we compute a constant $C_{\Lambda}$ so that

$$
\left\|\Lambda_{k}^{-1}\right\|_{\infty} \leq C_{\Lambda} \quad \forall k \geq M .
$$

Since $T(\bar{x})-\bar{x}=A f(\bar{x})$, we define

$$
Y_{k}:=\left\{\begin{array}{ll}
\left|\left[A^{(m)} f^{m}(\bar{x})\right]_{k}\right|, & k \leq m \\
\left|\Lambda_{k}^{-1} f_{k}(\bar{x})\right| & m+1 \leq k \leq M-1
\end{array} .\right.
$$


In order to construct the bound $Z_{k}$, we introduce the operator

$$
\left(J^{\dagger} x\right)_{k}:= \begin{cases}\left(D f^{(m)} x^{m}\right)_{k}, & k \leq m \\ \Lambda_{k} x_{k}, & k>m\end{cases}
$$

and consider the splitting

$$
\begin{aligned}
D T\left(\bar{x}+b_{1}\right) b_{2} & =\left[I-A D f\left(\bar{x}+b_{1}\right)\right] b_{2} \\
& =\left[I-A J^{\dagger}\right] b_{2}-A\left[D f\left(\bar{x}+b_{1}\right)-J^{\dagger}\right] b_{2}
\end{aligned} .
$$

Since $b_{1}, b_{2} \in B(r)$, it is convenient to write $b_{1}=r u, b_{2}=r v$, with $u, v \in B(1)$ and from the previous formula we have

$$
\left|[D T(\bar{x}+r u) r v]_{k}\right| \leq_{c w}\left|\left[\left(I-A J^{\dagger}\right) r v\right]_{k}\right|+\left|\left[A\left(D f(\bar{x}+r u)-J^{\dagger}\right) r v\right]_{k}\right| .
$$

Let $Z^{0}$ be defined as

$$
\left(Z^{0}\right)_{k}= \begin{cases}{\left[\left|I-A^{m} D f^{(m)}\right|\left\{w_{j}^{-s}\right\}_{j \leq m}\right]_{k},} & k \leq m \\ 0, & k>m\end{cases}
$$

so that $\left|\left[\left(I-A J^{\dagger}\right) r v\right]_{k}\right| \leq Z_{k}^{0} r$.

According with the degree $p$ of nonlinearity of the function $f(x)$, we can expand $\left[\left(D f(\bar{x}+r u)-J^{\dagger}\right) r v\right]_{k}$ as a polynomial in $r$

$$
\left[\left(D f(\bar{x}+r u)-J^{\dagger}\right) r v\right]_{k}=\sum_{i=1, \ldots, p} c_{k, i} r^{i}
$$

and we define the bounds $Z_{k}^{i}$ so that $Z_{k}^{i} \geq\left|c_{k, i}\right|$ uniformly in $u, v \in B(1)$. Finally the bound $Z_{k}$ is given by

$$
Z_{k}:=\left\{\begin{array}{ll}
{\left[\left|A^{m}\right|\left(\left(Z^{1}\right)^{m} r+\left(Z^{2}\right)^{m} r^{2}+\cdots+\left(Z^{p}\right)^{m} r^{p}\right]_{k}+Z_{k}^{0} r\right.} & k \leq m \\
\left|\Lambda_{k}^{-1}\right|\left(Z_{k}^{1} r+Z_{k}^{2} r^{2} \cdots+Z_{k}^{p} r^{p}\right) & m+1 \leq k<M
\end{array} .\right.
$$

Here $\left(Z^{i}\right)^{m}=\Pi_{m}^{X}\left(Z^{i}\right)$, which is the vector with the components $Z_{k}^{i}$ for $k \leq m$.

The definition of the tail bounds $Y_{M}$ and $Z_{M}$ satisfying (25) follows from uniform estimates, up to $w_{k}^{-s}$, of $\left|f_{k}(\bar{x})\right|$ and $\left|c_{k, i}\right|$ for $k \geq M$, where we assume to have found $f_{M}, Z_{M}^{i}$ such that

$$
\left|f_{k}(\bar{x})\right|_{\infty} \leq \frac{1}{w_{k}^{s}} f_{M}, \quad\left|c_{k, i}\right|_{\infty} \leq \frac{1}{w_{k}^{s}} Z_{M}^{i}, \quad \forall k \geq M, \quad \forall i=1, \ldots, p .
$$

Then, in view of (27), we define

$$
Y_{M}:=C_{\Lambda} f_{M} \quad Z_{M}:=C_{\Lambda}\left(Z_{M}^{1} r+\cdots+Z_{M}^{p} r^{p}\right) .
$$

We remark that the definition of the vector $Y$ and $Z$ is based on a combination of rigorous computations and analytical estimates: utilizing rigorous computation ensures that the rounding errors are controlled whenever a computation is performed; analytical estimates control the truncation errors arising from the finite-dimensional approximation. In particular, analytical estimates will be necessary to control $f_{k}(x)$ for $k \geq M$ and to bound the coefficients $c_{k, i}$ appearing in (32), both for each $k<M$ and uniformly for $k \geq M$.

\section{Application to NLS}

We now apply the computational technique described in the previous section to the control problem of Section 2.1. As mentioned in the introduction, the goal is to prove conditions (A) and (B) for all 
eigenvalue of the linearized NLS. To check whether the $\Gamma_{n}$ are non-zero, we first have to rigorously compute the eigenvalues and the eigenfunction of $\mathcal{L}$, i.e. the solutions of system $(19 \mathrm{c}),(19 \mathrm{~d})$. Since the linearization depends on the solution $\phi(x)$ of the Schrödinger equation, the bound state $\phi(x)$ has to be rigorously computed as well. Hence we perform three computations, each one using rigorous numerics.

i) For a choice of $\mu>0$ and $\sigma \in\{ \pm 1\}$, we compute the solution $\phi(x)$ for $5 \mathrm{a}$, , 5b];

ii) Given the state $\phi(x)$, we compute the eigenpairs $(\beta, W)$ by solving $119 \mathrm{c}, 119 \mathrm{~d})$ and check that $\Gamma_{n}$ is different from zero;

iii) We prove that the computed eigenvalues are simple.

For each of these problems we state the nonlinear map $f(x)$, the Banach space $X^{s}$, the Jacobian $D f^{(m)}$, and some of the necessary analytic estimates. However, in order to increase readability, we delegate most of the analytical estimates and the technical details to the Appendix.

\subsection{Computing the bound states $\phi(x)$}

Bound-states $\phi$ are solutions of the BVP

$$
\left\{\begin{array}{l}
-\phi^{\prime \prime}+\sigma \mu \phi-\sigma \phi^{3}=0, \quad x \in(0,1) \\
\phi(0)=\phi(1)=0
\end{array} .\right.
$$

Expanding $\phi$ w.r.t the sine-basis $\{\sqrt{2} \sin (\pi n x)\}_{n \geq 1}$ gives

$$
\phi(x)=\sqrt{2} \sum_{n \geq 1} \alpha_{n} \sin (\pi n x), \quad \alpha_{n} \in \mathbb{R} .
$$

Using the symmetry of the sine functions, this expansion is equivalent to

$$
\phi(x)=\sqrt{2} \sum_{n \in \mathbb{Z}} b_{n} \sin (\pi n x), \quad b_{n} \in \mathbb{R},
$$

where the coefficients satisfy $b_{-n}=-b_{n}$. This is readily seen by defining $\alpha_{n}=2 b_{n}$. The advantage of this representation is that the projection of the cubic term onto the basis elements $\sqrt{2} \sin (\pi n x)$ takes the simple form

$$
<\phi^{3}, \sqrt{2} \sin (\pi n \bullet)>=-4 \sum_{\substack{p+k+\ell=n \\ p, k, \ell \in \mathbb{Z}}} b_{p} b_{k} b_{\ell} ;
$$

see Appendix A. Inserting (36), 37) into system (35) and using $b_{-n}=-b_{n}$, we obtain the infinitedimensional algebraic system

$$
f(b)=\left(f_{1}, f_{2}, \ldots, f_{n}, \ldots\right)(b)=0, \quad n \geq 1
$$

for the unknown $b=\left\{b_{n}\right\}_{n \geq 0}$, where

$$
f_{n}(b)=\left(\pi^{2} n^{2}+\sigma \mu\right) b_{n}+2 \sigma \sum_{\substack{p+k+\ell=n \\ p, k, \ell \in \mathbb{Z}}} b_{p} b_{k} b_{\ell} \quad n \geq 1 .
$$

Note that we only considered $n \geq 1$ : by the symmetry of the $b_{k}$ 's we have that $f_{-n}(b)=-f_{n}(b)$. Since the unknowns are $b_{k}$ with $k \geq 1$ ( $b_{0}$ may be set equal to zero), it is sufficient to solve $f_{n}(b)=0$ for $n \geq 1$. 


\subsubsection{Ground State and even exited states}

The ground state and the exited states with an even number of nodal points are functions that are symmetric with respect to $x=\frac{1}{2}$. This means that the even Fourier coefficients vanish, i.e. $\alpha_{2 n}=0$ and $b_{2 n}=0$, as well as $f_{2 n}(b)=0$. Hence we discard the even Fourier coefficients and we introduce the sequence of odd coefficients

$$
b_{n}^{o}=b_{2 n-1} .
$$

The symmetry conditions for the new sequence read $b_{0}^{o}=-b_{1}^{o}$ and $b_{-n}^{o}=-b_{n+1}^{o}$ for $n \geq 1$. Similarly, we discard the even component of $\left\{f_{n}\right\}$ and introduce the reduced system $f_{n}^{o}=f_{2 n-1}$ for $n \geq 1$. In terms of the unknown $b^{o}=\left\{b_{n}^{o}\right\}_{n \geq 1}$ the new system reads

$$
f_{n}^{o}\left(b^{o}\right)=\left(\pi^{2}(2 n-1)^{2}+\sigma \mu\right) b_{n}^{o}+2 \sigma \sum_{\substack{p+k+\ell=n+1 \\ p, k, \ell \in \mathbb{Z}}} b_{p}^{o} b_{k}^{o} b_{\ell}^{o} .
$$

We wish to bound the solution $b^{o}=\left\{b_{k}^{o}\right\}_{k \geq 1}$ of $f^{o}\left(b^{o}\right)=0$. For the remainder of this section we omit the superscript $(\cdot)^{o}$. We look for the solution in the Banach space

$$
X^{s}=\left\{b=\left\{b_{k}\right\}_{k \geq 1}, b_{k} \in \mathbb{R}:\|b\|_{s}<\infty\right\}
$$

for $s \geq 2$. Note that $f: X^{s} \rightarrow X^{s-2}$.

Suppose that the finite-dimensional parameter $m$ has been chosen and that a numerical solution $\bar{b}=\left\{\bar{b}_{1}, \bar{b}_{2}, \ldots, \bar{b}_{m}\right\}$ of $f^{(m)}(b)=0$ has been computed. (A package such as Maple may conveniently be used to determine the Fourier coefficients of the elliptic functions up to a desired accuracy.) By direct computation, the Jacobian of $f^{(m)}$ and the coefficients $\Lambda_{n}$ are given by

$$
\frac{\partial f_{n}}{\partial b_{j}}(\bar{b})=\left(\pi^{2}(2 n-1)^{2}+\sigma \mu\right) \delta_{n-j}+6 \sigma\left[\sum_{k_{1}+k_{2}=n-j+1} \bar{b}_{k_{1}} \bar{b}_{k_{2}}-\sum_{k_{1}+k_{2}=n+j} \bar{b}_{k_{1}} \bar{b}_{k_{2}}\right], \quad k_{1}, k_{2} \in \mathbb{Z}
$$

and

$$
\Lambda_{n}:=\pi^{2}(2 n-1)^{2}+\sigma \mu, \quad n>m,
$$

respectively. We now introduce the operator $T$ according to 22, 23). (In the de-focusing case $\sigma=-1$ the parameter $m$ must be such that $\pi^{2}(2 m-1)^{2}>\sigma \mu$ to ensure the invertibility of $\Lambda_{n}$ and, by extension, of $A$ ). Note that the Jacobian is symmetric, as expected from the variational nature of the problem.

For the construction of the radii polynomials we fix the computational parameter $M=3 \mathrm{~m}$ and set

$$
C_{\Lambda}=\left(\pi^{2}(2 M-1)^{2}+\sigma \mu\right)^{-1} .
$$

The choice of $M$ is motivated by the fact that $f_{n}(\bar{b})=0(\forall n \geq 3 m)$, which allows us to set $Y_{M}:=0$. The definition of the vector $Y=\left(Y_{1}, \ldots, Y_{M-1}\right)$ is given by 28 , while the vector $Z=\left(Z_{1}, \ldots, Z_{M-1}\right)$ and the tail bound $Z_{M}$ follow from careful estimates of the coefficients $c_{k, i}$, given in terms of convolution products. We use the estimates provided in the paper [18, where sharp bounds for the convolution products are proved. In Appendix A we list some of required analytical estimates and the definition of the remaining bounds $Z_{k}, Z_{M}$; see [18] for details.

\subsubsection{Odd exited states}

The procedure for the computation of the odd exited states (i.e. solutions $\phi(x)$ with an odd number of nodal points) is similar to the one discussed in the previous section. Since the solutions are odd w.r.t. $x=\frac{1}{2}$, only the even Fourier coefficients have to be computed. Consequently, we introduce the vector of unknowns $b_{n}^{e}=b_{2 n}$ and the system

$$
f_{n}^{e}\left(b^{e}\right)=\left(\pi^{2}(2 n)^{2}+\sigma \mu\right) b_{n}^{e}+2 \sigma \sum_{\substack{p+k+\ell=n \\ p, k, \ell \in \mathbb{Z}}} b_{p}^{e} b_{k}^{e} b_{\ell}^{e}
$$


to be solved for $n \geq 1$. Note that in this case the Jacobian is given by

$$
\frac{\partial f_{n}^{e}}{\partial b_{j}^{e}}(\bar{b})=\left(\pi^{2}(2 n)^{2}+\mu\right) \delta_{n-j}+6\left[\sum_{k_{1}+k_{2}=n-j} \bar{b}_{k_{1}} \bar{b}_{k_{2}}-\sum_{k_{1}+k_{2}=n+j} \bar{b}_{k_{1}} \bar{b}_{k_{2}}\right], \quad k_{1}, k_{2} \in \mathbb{Z} .
$$

The definition of the fixed point operator $T$ and the construction of the radii polynomials are similar, mutatis mutandis, to the previous case.

Remark 3 For clarity, we explicitly show what the enclosure of the sequence $b^{o}$ or $b^{e}$ means for the actual Fourier coefficients $b_{n}$ in (36). Depending on the symmetry of the state $\phi(x)$, denote by $b_{n}^{*}$ the odd $b_{n}^{o}$ or the even $b_{n}^{e}$ coefficients.

Suppose that, for a finite-dimensional parameter $m=m_{\phi}$ and a decay rate $s=s_{\phi}>2$, the computational method results in the enclosure radius $r=r_{\phi}$. This means that the sequence $b^{*}=\left\{b_{n}^{*}\right\}_{n \geq 1}$ satisfies

$$
\left|b_{n}^{*}-\bar{b}_{n}^{*}\right| \leq r_{\phi} / w_{n}^{s_{\phi}} \text { for } n=1, \ldots, m_{\phi}, \quad \text { and } \quad\left|b_{n}^{*}\right| \leq r_{\phi} / w_{n}^{s_{\phi}} \text { for } n>m_{\phi} .
$$

Hence, the sequence $b=\left\{b_{n}\right\}$ satisfies

$$
\left|b_{n}-\bar{b}_{n}\right| \leq r_{\phi} \frac{1}{\left.w_{\left[\frac{n}{2}\right]}^{s_{\phi}}\right]} \text { for }|n| \leq 2 m_{\phi}, \quad \text { and } \quad\left|b_{n}\right| \leq r_{\phi} \frac{1}{w_{\left[\frac{n}{2}\right]}^{s_{\phi}}} \text { for }|n|>2 m_{\phi}
$$

where the odd or even terms of $\bar{b}$ are equal to $\bar{b}^{*}$ and the others are set to zero.

Remark 4 For the remainder of the paper the subscripted constants $s_{\phi}, m_{\phi}, M_{\phi}$, and $r_{\phi}$ introduced in the previous remark will be kept fixed. As indicated, they refer to the parameters associated with the computation of the bound states. In the next section (see eq. (44)) a new set of parameters s, $m, M$ will be chosen for the computation of the eigenvalues and eigenfunctions of the linearization. The purpose of adopting the subscript notation for $s_{\phi}, m_{\phi}$ etc. is to avoid confusion of the two sets of parameters.

\subsection{Solving the eigenvalue problem}

Once the solution $\phi(x)$ is computed, the eigenvalue problem consists in solving $(19 \mathrm{c}),(19 \mathrm{~d})$ for the unknowns $\beta, w(x), z(x)$ As before, we expand $w(x)$ and $z(x)$ w.r.t. the Fourier-sine basis

$$
w(x)=\sqrt{2} \sum_{n \geq 1} c_{n} \sin (\pi n x), \quad z(x)=\sqrt{2} \sum_{n \geq 1} d_{n} \sin (\pi n x), \quad c_{n}, d_{n} \in \mathbb{C},
$$

so we obtain the infinite-dimensional algebraic system

$$
f=\left(f_{1}, f_{2} \ldots\right)=0, \quad f_{n}=\left[\begin{array}{c}
(\pi n)^{2} c_{n}+(\sigma \mu-\beta) c_{n}-\sigma \sum_{\ell \geq 1} F_{n, \ell}\left(2 c_{\ell}-d_{\ell}\right) \\
(\pi n)^{2} d_{n}+(\sigma \mu+\beta) d_{n}+\sigma \sum_{\ell \geq 1} F_{n, \ell}\left(c_{\ell}-2 d_{\ell}\right)
\end{array}\right],
$$

to be solved for unknowns $\left(\beta, c_{1}, c_{2}, \ldots, d_{1}, d_{2}, \ldots\right)$. The matrix $F=\left\{F_{n, \ell}\right\}$ corresponds to the term $\phi(x)^{2}$ and it is given explicitly by

$$
F_{n, \ell}=2\left(\sum_{p+k=n+\ell} b_{k} b_{p}-\sum_{p+k=n-\ell} b_{k} b_{p}\right)
$$

where $b_{n}$ are the coefficients in (36); see Appendix B. Since the system is invariant under rescaling of eigenfunctions, we need to choose a normalization to obtain isolated solutions. Rather than introducing a new equation, we remove one of the unknowns. Assume that we have computed a numerical solution $\hat{x}=\left(\bar{\beta},\left\{\bar{c}_{k}, \bar{d}_{k}\right\}_{k=1}^{m}\right)$ of the system 42 (for $\left.n=1, \ldots, m\right)$ and that $\bar{c}_{j_{*}}$ is the 
largest value of the $\bar{c}_{k}$ 's. Then we fix the value of $c_{j_{*}}=\bar{c}_{j_{*}}$ and we remove $c_{j_{*}}$ from the unknowns. The reduced vector of unknowns is

$$
x=\left(\beta, d_{1}, c_{1}, d_{1}, \ldots, c_{j_{*}-1}, d_{j_{*}-1}, d_{j_{*}}, c_{j_{*}+1}, d_{j_{*}+1}, \ldots\right)
$$

and, grouping $c_{k}, d_{k}$, we write

$$
x=\left(x_{0}, x_{1}, x_{2}, \ldots\right), \quad x_{0}=\beta, x_{j_{*}}=\left(d_{j_{*}}\right), x_{k}=\left(c_{k}, d_{k}\right), k \neq j_{*} .
$$

Now choose a decay rate $s$, finite-dimensional parameter $m$, and computational parameter $M$ so that

$$
s<s_{\phi}, \quad m=3 m_{\phi}, \quad M>m+4 m_{\phi} .
$$

Then the $s$-norm of $x$ and the corresponding Banach space are defined by

$$
\|x\|_{s}=\sup \left\{|\beta|,\left|d_{j_{*}}\right| j_{*}^{s}, \sup _{k \geq 1, k \neq j_{*}}\left\{\left|c_{k}\right| k^{s},\left|d_{k}\right| k^{s}\right\}\right\}
$$

and

$$
X^{s}:=\left\{x:\|x\|_{s}<\infty\right\},
$$

respectively. Keeping in mind that $c_{j_{*}}$ is fixed, we look for a zero of

$$
f(x)=\left(f_{1}, f_{2}, \ldots\right): X^{s} \rightarrow W=X^{s-2}
$$

for $s \geq 2$ and $f_{n}$ as in 42 . Let

$$
\bar{x}=\left(\bar{\beta}, \bar{d}_{j_{*}},\left\{\bar{c}_{k}, \bar{d}_{k}\right\}_{k \geq q, \neq j_{*}}^{m}\right)
$$

be an approximate zero for $f^{(m)}(x)$ (which can be obtained by simply removing $\bar{c}_{j_{*}}$ from $\hat{x}$ ). The Jacobian $D f^{(m)}=\frac{\partial f^{(m)}}{\partial x^{(m)}}(\bar{x})$ is given by

$$
D f^{(m)}=\left[\begin{array}{c|cc|c|c|c|cc}
\frac{\partial}{\partial \beta} & \frac{\partial}{\partial c_{1}} & \frac{\partial}{\partial d_{1}} & \ldots & \frac{\partial}{\partial d_{j_{*}}} & \ldots & \frac{\partial}{\partial c_{m}} & \frac{\partial}{\partial d_{m}}
\end{array}\right],
$$

where

$$
\frac{\partial}{\partial \beta}=\left[\begin{array}{c}
-\bar{c}_{1} \\
\bar{d}_{1} \\
-\bar{c}_{2} \\
\bar{d}_{2} \\
\vdots \\
-\bar{c}_{m} \\
\bar{d}_{m}
\end{array}\right] \quad \frac{\partial}{\partial c_{j}}, \frac{\partial}{\partial d_{j}}=\left[\begin{array}{cc}
-2 \sigma F_{1, j} & \sigma F_{1, j} \\
\sigma F_{1, j} & -2 \sigma F_{1, j} \\
\vdots & \vdots \\
\pi^{2} j^{2}+\sigma \mu-\bar{\beta}-2 \sigma F_{j, j} & \sigma F_{j, j} \\
\sigma F_{j, j} & \pi^{2} j^{2}+\sigma \mu+\bar{\beta}-2 \sigma F_{j, j} \\
\vdots & \vdots \\
-2 \sigma F_{m, j} & \sigma F_{m, j} \\
\sigma F_{m, j} & -2 \sigma F_{m, j}
\end{array}\right]
$$

and, for $k>m$,

$$
\Lambda_{k}=\frac{\partial F_{k}}{\partial\left(c_{k}, d_{k}\right)}(\bar{x})=\left[\begin{array}{cc}
\pi^{2} k^{2}+\sigma \mu-\bar{\beta}-2 \sigma F_{k, k} & \sigma F_{k, k} \\
\sigma F_{k, k} & \pi^{2} k^{2}+\sigma \mu+\bar{\beta}-2 \sigma F_{k, k}
\end{array}\right] .
$$


Hence, according to 22, 23 , the operator $A$ is defined by the infinite-dimensional matrix

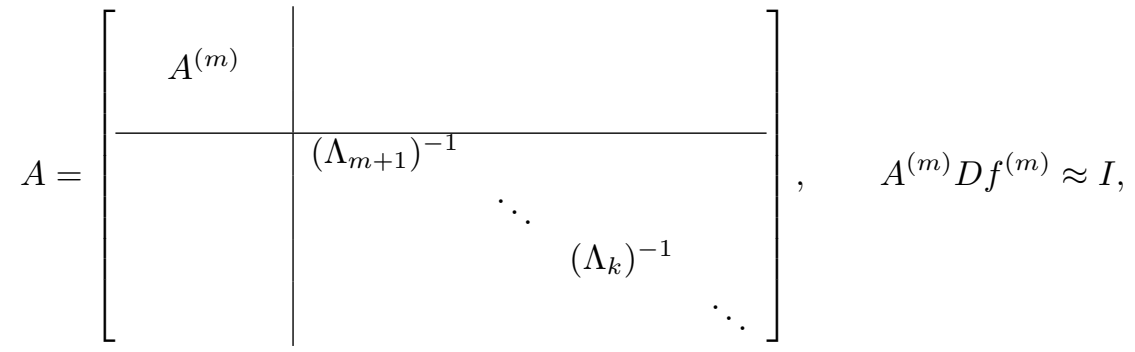

and the operator $T: X \rightarrow X$ is given by

$$
T(x)=x-A f(x) .
$$

The operator $T$ is well-defined, since the operator $A$ maps $X^{s-2}$ to $X^{s}$ and is invertible. These properties follow from the behaviour of $\Lambda_{k}^{-1}$ for $k>m$; in Appendix B we prove that, for sufficiently large $m$, there exists a constant $\mathcal{C}_{\Lambda}(m)$ such that

$$
\left\|\Lambda_{k}^{-1}\right\|_{\infty} \leq \frac{\mathcal{C}_{\Lambda}(m)}{k^{2}} \quad(\forall k>m)
$$

Hence, fixed points of $T$ correspond to zeros of $f(x)$.

\subsubsection{Construction of the bounds $\mathrm{Y}, \mathrm{Z}$}

In deriving rigorous bounds, the most difficult terms are not actually the nonlinear ones (given by the product $\beta c_{k}$ and $\left.\beta d_{k}\right)$, but the linear terms, such as $\sum_{\ell \geq 1} F_{n, \ell}\left(2 c_{\ell}-d_{\ell}\right)$. This is because each $F_{n, \ell}$ is defined as a convolution of $b_{k}$ 's; the latter, however, are the result of the prior computation of $\phi(x)$ and, as such, are only known to lie in certain intervals. Therefore, in order to design a successful scheme, we need to find sharp estimates for the terms $F_{n, \ell}$.

Using the notation of Remarks 3 and 4 , define

$$
\begin{gathered}
\mathcal{E}(q)=4^{s_{\phi}} r_{\phi}^{2} \frac{\alpha_{q}^{(2)}}{w_{q}^{s_{\phi}}}+2 r_{\phi} 2^{s_{\phi}} \sum_{j=-2 m_{\phi}}^{2 m_{\phi}}\left|\bar{b}_{j}\right| w_{q-j}^{-s_{\phi}} \\
\tilde{\mathcal{E}}(q)=w_{q}^{s_{\phi}} \mathcal{E}(q),
\end{gathered}
$$

where $\alpha_{q}^{(2)}$ is defined in eq. (55) of the appendix (note that the constant $M$ in (55) is to be interpreted as $M_{\phi}$ ).

Lemma 3 For any $q$

$$
\left|\sum_{p+k=q} b_{k} b_{p}\right| \leq\left|\sum_{p+k=q} \bar{b}_{p} \bar{b}_{k}\right|+\mathcal{E}(q)
$$

In particular, for $|q| \geq 4 m_{\phi}$

$$
\left|\sum_{p+k=q} b_{k} b_{p}\right| \leq \mathcal{E}(q)
$$

Proof. See Appendix B. 
Remark $5 \mathcal{E}(-q)=\mathcal{E}(q)$ and $\tilde{\mathcal{E}}(-q)=\tilde{\mathcal{E}}(q)$. The functions $\mathcal{E}(q)$ and $\tilde{\mathcal{E}}(q)$ are decreasing in $q$, for $q \geq m=3 m_{\phi}$.

Defining

$$
\begin{gathered}
b_{\max }:=\max _{n}\left|\bar{b}_{n}\right| \\
\left|\bar{F}_{n, \ell}\right|:=2\left(\left|\sum_{p_{1}+p_{2}=n+\ell} \bar{b}_{p_{1}} \bar{b}_{p_{2}}\right|+\left|\sum_{p_{1}+p_{2}=n-\ell} \bar{b}_{p_{1}} \bar{b}_{p_{2}}\right|\right)
\end{gathered}
$$

we list some properties of $F=\left\{F_{n, \ell}\right\}$.

Lemma 4 1. F is symmetric.

2. $F_{n, \ell}=0$ for $n+\ell=0$.

3. For any $n, \ell \geq 1$

$$
F_{n, \ell} \in 2\left[\sum_{|k| \leq 2 m_{\phi}} b_{k}\left(b_{n+\ell-k}-b_{n-\ell-k}\right)\right] \pm 8 r_{\phi}\left(b_{\max }+r_{\phi}\right) \frac{2^{s_{\phi}}}{\left(s_{\phi}-1\right) m_{\phi}^{s_{\phi}-1}} .
$$

4. For any $n, \ell \geq 1$

$$
\left|F_{n, \ell}\right| \leq\left|\bar{F}_{k, \ell}\right|+2 \mathcal{E}(n+\ell)+2 \mathcal{E}(n-\ell) .
$$

5. For any $n, \ell \geq 1$ such that $|n-\ell|>4 m_{\phi}$

$$
\left|F_{n, \ell}\right| \leq \frac{2}{(n+\ell)^{s_{\phi}}} \tilde{\mathcal{E}}(n+\ell)+\frac{2}{(n-\ell)^{s_{\phi}}} \tilde{\mathcal{E}}(n-\ell) .
$$

Proof. 1. Follows directly from (43).

2. Immediate consequence of the fact that the even or the odd elements of $\left\{b_{n}\right\}$ are zero.

3 .

$$
F_{n, \ell}=2\left[\sum_{|k| \leq 2 m_{\phi}} b_{k}\left(b_{n+\ell-k}-b_{n-\ell-k}\right)\right]+2\left[\sum_{|k|>2 m_{\phi}} b_{k}\left(b_{n+\ell-k}-b_{n-\ell-k}\right)\right]
$$

and so

$$
\left|F_{n, \ell}-2 \sum_{|k| \leq 2 m_{\phi}} b_{k}\left(b_{n+\ell-k}-b_{n-\ell-k}\right)\right| \leq 2 \sum_{|k|>2 m_{\phi}}\left|b_{k}\right|\left(\left|b_{n+\ell-k}\right|+\left|b_{n-\ell-k}\right|\right) .
$$

From (41) it follows $\left|b_{n}\right| \leq b_{\max }+r_{\phi}(\forall n)$, hence the right hand side of the previous inequality can be bounded by $2\left(2\left(b_{\max }+r_{\phi}\right) 2^{s^{\phi}} r_{\phi} \sum_{|k|>m_{\phi}} 1 /|k|^{s_{\phi}}\right) \leq 8\left(b_{\max }+r_{\phi}\right) r_{\phi} \frac{2^{s_{\phi}}}{\left(s_{\phi}-1\right) m_{\phi}^{s_{\phi}-1}}$.

4. Combine (43) and (48).

5. Since $\bar{b}_{n}=0$ for $|n| \geq 2 m_{\phi}$, the estimates follows from 49 and the definition of $\tilde{\mathcal{E}}(q)$.

We define a constant $C_{\Lambda}$ satisfying (27) by

$$
C_{\Lambda}=\frac{\mathcal{C}_{\Lambda}(M)}{M^{2}},
$$

where $\mathcal{C}_{\Lambda}(M)$ has been introduced in 47 .

The bounds $Y_{k}$ for $k=1, \ldots, m$ are defined as in 28). The next lemma provides a uniform bound for the tail part of $f(\bar{x})$. 
Lemma 5 Let $\mathcal{H}=\mathcal{H}(M)$ be the vector

$$
\mathcal{H}:=2 \sum_{1 \leq \ell \leq m}\left(\tilde{\mathcal{E}}(M+\ell)+\frac{1}{\left(1-\frac{\ell}{M}\right)^{s_{\phi}}} \tilde{\mathcal{E}}(M-\ell)\right)\left[\begin{array}{r}
\left|\left(2 \bar{c}_{\ell}-\bar{d}_{\ell}\right)\right| \\
\left.\mid \bar{c}_{\ell}-2 \bar{d}_{\ell}\right) \mid
\end{array}\right] .
$$

Then $|f(\bar{x})| \leq \frac{1}{k^{s} \phi} \mathcal{H}(M)$ for all $k \geq M$.

Proof. See Appendix B.

Since $s<s_{\phi}$, the previous lemma implies that $\left|f_{k}(\bar{x})\right| \leq \frac{1}{w_{k}^{s}} \frac{\mathcal{H}(M)}{M^{s} \phi^{-s}}$. We therefore set

$$
Y_{M}:=C_{\Lambda} \frac{|\mathcal{H}(M)|_{\infty}}{M^{s_{\phi}}-s} .
$$

As for the definition of the bounds $Z_{k}$ and $Z_{M}$, it is convenient to have a formula for the coefficients $c_{k, i}$ defined in 32 . To this end, we write $D f(\bar{x}+r u)-J^{\dagger}=$

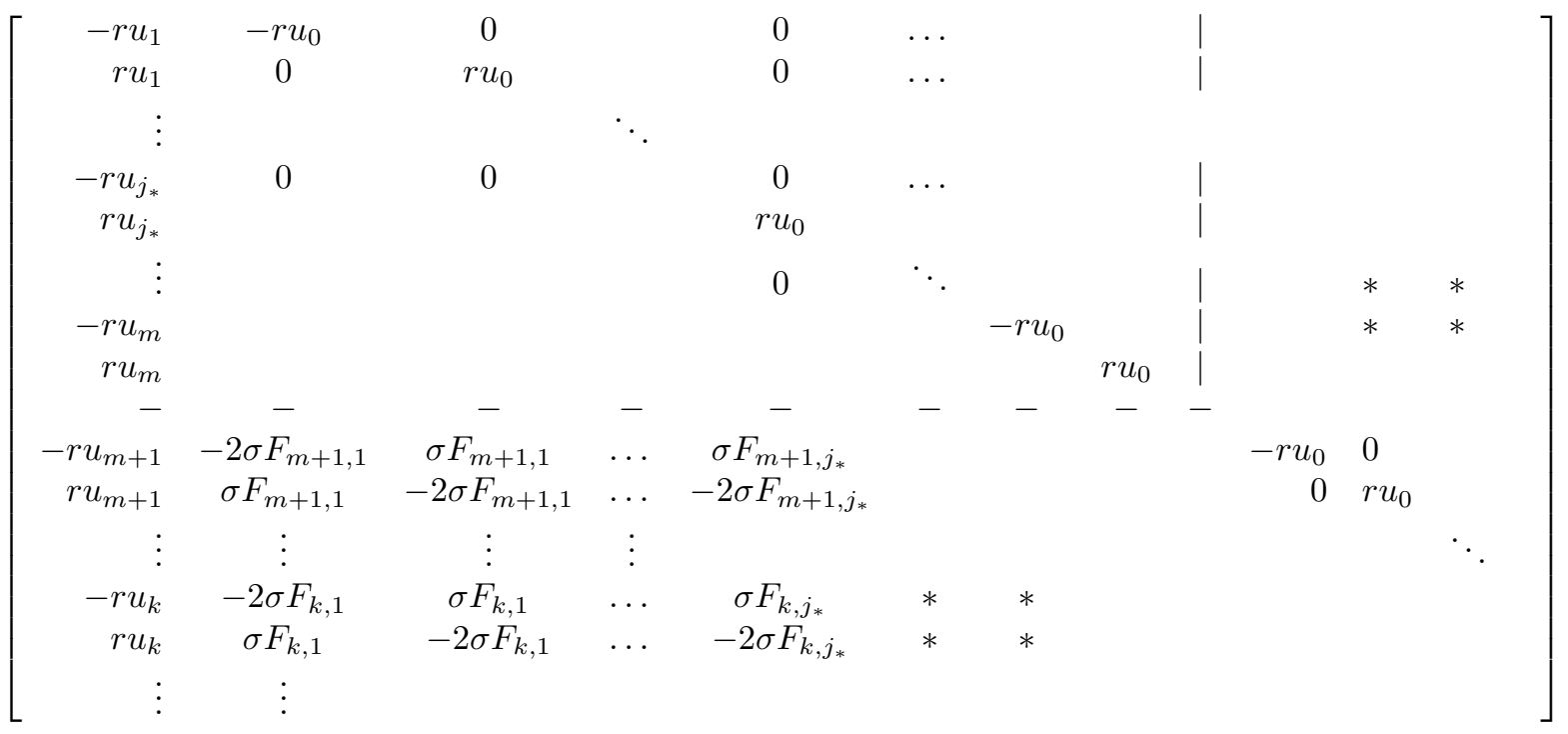

where the $(k, j)$-block is

$$
\begin{array}{cc}
* & * \\
* & *
\end{array}=\sigma\left[\begin{array}{cc}
-2 F_{k, j} & F_{k, j} \\
F_{k, j} & -2 F_{k, j}
\end{array}\right] \text {. }
$$


Thus we have

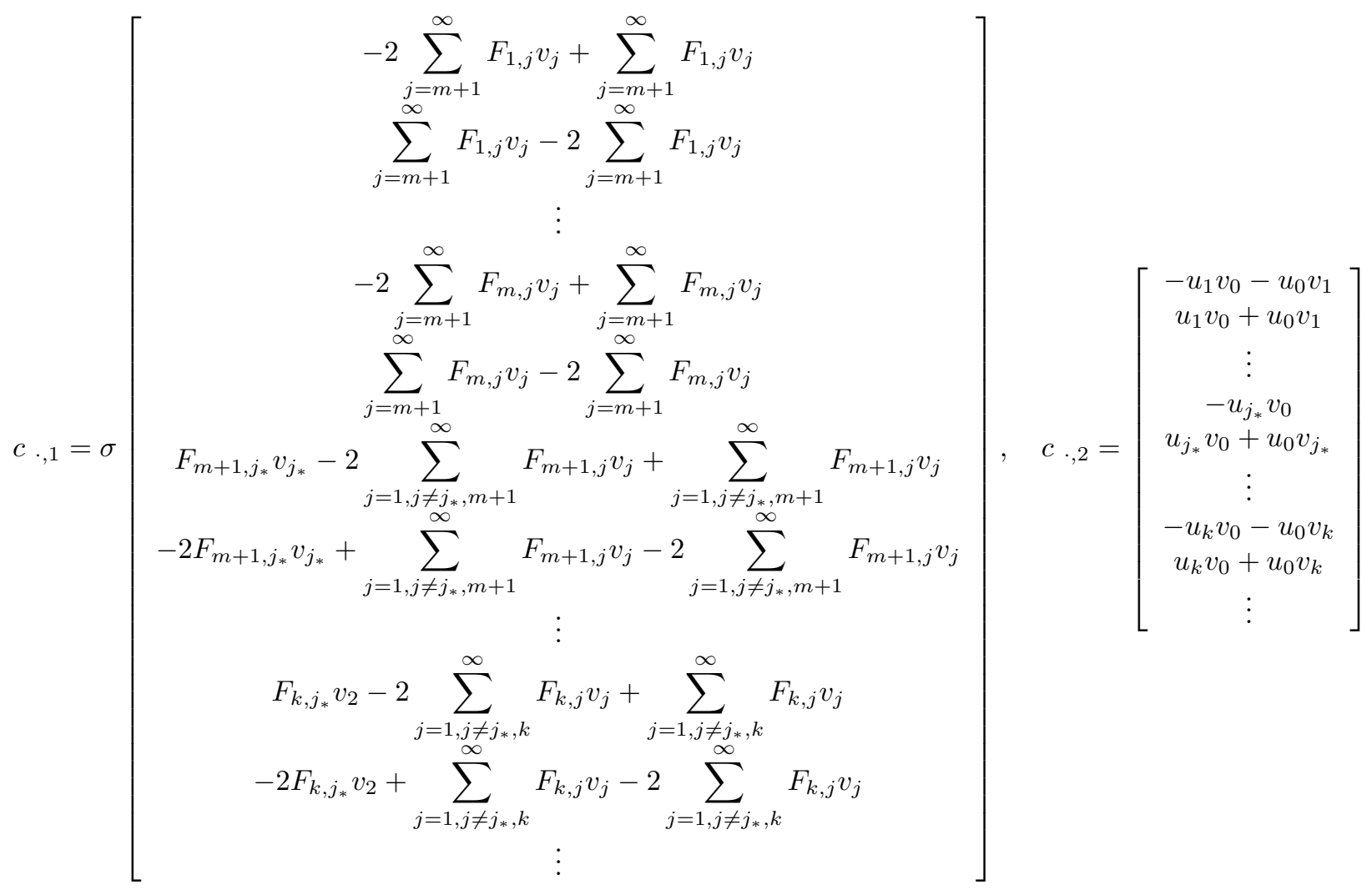

Since $\left|u_{j}\right|,\left|v_{j}\right| \leq j^{-s}$, we obtain the estimates

$$
\left|c_{k, 1}\right| \leq\left[\begin{array}{c}
3 \sum_{j=m+1}^{\infty}\left|F_{k, j}\right| j^{-s} \\
3 \sum_{j=m+1}^{\infty}\left|F_{k, j}\right| j^{-s}
\end{array}\right] k=1, \ldots, m, \quad\left|c_{k, 1}\right| \leq\left[\begin{array}{c}
3 \sum_{j=1, j \neq k}^{\infty}\left|F_{k, j}\right| j^{-s}-2\left|F_{k, j_{*}}\right| j_{*}^{-s} \\
3 \sum_{j=1, j \neq k}^{\infty}\left|F_{k, j}\right| j^{-s}-\left|F_{k, j_{*}}\right| j_{*}^{-s}
\end{array}\right] k \geq m+1
$$

and

$$
\left|c_{j_{*}, 2}\right| \leq j_{*}^{-s}\left[\begin{array}{l}
2 \\
4
\end{array}\right] \quad\left|c_{k, 2}\right| \leq 4 k^{-s}\left[\begin{array}{l}
1 \\
1
\end{array}\right] \quad k \geq 1, k \neq j_{*},
$$

where we used the fact that $\left|z_{1} z_{2}\right|=\max \left\{\mid \operatorname{Re}\left(z_{1} z_{2}|,| \operatorname{Im}\left(z_{1} z_{2} \mid\right\} \leq 2\left|z_{1}\right|\left|z_{2}\right|\right.\right.$.

Note that the vectors $c_{k, 1}$ are given as series. We can provide a bound using formula 499.

Define

$$
H^{1}(k):=3 \begin{cases}\sum_{j=m+1}^{4 m_{\phi}+k}\left|\bar{F}_{k, j}\right| j^{-s}+2 \frac{\mathcal{E}(m+1+k)+\mathcal{E}(m+1-k)}{(s-1) m^{s-1}} & k \leq m \\ \sum_{j=\max \left\{1, k-4 m_{\phi}\right\}, j \neq k}^{k+4 m_{\phi}}\left|\bar{F}_{k, j}\right| j^{-s}+2 \sum_{j=1}^{k-1} \mathcal{E}(j-k) j^{-s} & k>m . \\ +\mathcal{E}(1) \frac{2}{(k+1)^{s}}+\mathcal{E}(2) \frac{2}{(s-1)(k+1)^{s-1}}+2 \mathcal{E}(k+1)+\mathcal{E}(k+2) \frac{2}{(s-1)} & \end{cases}
$$

Then we have 


\section{Lemma 6}

$$
\left|c_{k, 1}\right|_{\infty} \leq H^{1}(k), \quad k \geq 1 .
$$

Proof. See Appendix B.

In view of Lemma 6 , we define $Z^{1}, Z^{2}$ as the vectors with components

$$
\begin{gathered}
Z_{k}^{1}=H^{1}(k)\left[\begin{array}{l}
1 \\
1
\end{array}\right], \quad k=1, \ldots, M-1, \\
Z_{k}^{2}=4 k^{-s}\left[\begin{array}{l}
1 \\
1
\end{array}\right] \text { for } k \neq j_{*}, Z_{j_{*}}^{2}=2 j_{*}^{-s}\left[\begin{array}{l}
1 \\
2
\end{array}\right], \quad k=1, \ldots, M-1 .
\end{gathered}
$$

The final pieces are the bounds $Z_{M}^{1}, Z_{M}^{2}$ satisfying (34) that will give the tail bound $Z_{M}$. Clearly, we can set $Z_{M}^{2}:=4$, while $Z_{M}^{1}$ has to be defined as a uniform bound (up to $w_{k}^{-s}$ ) of $H^{1}(k)$ for $k \geq M$.

Lemma 7 Define

$Z_{M}^{1}:=6\left[\sum_{p=1}^{4 m_{\phi}}\left(\left|\sum_{p_{1}+p_{2}=p} \bar{b}_{p_{1}} \bar{b}_{p_{2}}\right| \cdot \frac{1}{\left(1-\frac{p}{M}\right)^{s}+1}\right)+\tilde{\mathcal{E}}(M+1)+\frac{\tilde{\mathcal{E}}(M+2)}{s-1}+\tilde{\mathcal{E}}(1) \gamma_{M}+\tilde{\mathcal{E}}(1)+\frac{\tilde{\mathcal{E}}(2)}{s-1}\right]$

where $\gamma_{k}$ is given in [54). Then $\left|c_{k, 1}\right|_{\infty} \leq \frac{1}{w_{k}^{s}} Z_{M}^{1}$, for all $k \geq M$.

Proof. See Appendix B.

\subsection{The eigenvalues are simple}

Let $\beta$ and $W=\left(\begin{array}{l}w \\ z\end{array}\right)$ be solution of the eigenvalue problem $19 \mathrm{c}, 119 \mathrm{~d}$ ). To show that $\beta$ is simple it will be verified that there is no eigenfunction $V$ of the operator $\mathcal{N}$ orthogonal to $W$. Define the operators $L_{\beta}$ and $G$ by $L_{\beta}(V)=(\mathcal{N}-\beta I) V$ and

$$
G\left(\lambda_{0}, V\right)=\left[\begin{array}{c}
<W, V> \\
\lambda_{0} W+L_{\beta}(V)
\end{array}\right], \quad \lambda_{0} \in \mathbb{R}, V \in C_{0}^{2}([0,1]),
$$

respectively.

Lemma 8 If $X_{0}=(0,0)$ is a locally unique solution of $G(X)=0$, then the eigenvalue $\beta$ is simple.

Proof. Assume that $\lambda_{0}=0, V=0$ is a locally unique solution, but that $\beta$ is not simple. Then there exists a function $\mathcal{V}$ such that $\langle W, \mathcal{V}\rangle=0$ and $L_{\beta}(\mathcal{V})=0$. However, this implies that $X_{\lambda}=(0, \lambda \mathcal{V})$ is a solution for every $\lambda \in \mathbb{R}$, so the zero-solution is not locally unique. Contradiction.

To apply the spectral method of Section 4.2 we recast $G\left(\lambda_{0}, V\right)=0$ as an infinite-dimensional algebraic system with unknowns $x=\left(\lambda_{0},\left\{c_{n}\right\},\left\{d_{n}\right\}\right)$. Suppose that $\left(\beta,\left\{c_{n}^{\prime}, d_{n}^{\prime}\right\}_{n \geq 1}\right)$ represents the eigenpair $(\beta, W)$, that is $f\left(\beta,\left\{c_{n}^{\prime}, d_{n}^{\prime}\right\}_{n \geq 1}\right)=0$, see 42$)$. Then we introduce the system

$$
g=\left(g_{0}, g_{1}, g_{2}, \ldots\right)(x)=0
$$

given by

$$
g_{0}=\sum_{n \geq 1}\left(c_{n}^{\prime} c_{n}+d_{n}^{\prime} d_{n}\right) \quad \text { and } \quad g_{n}=\lambda_{0}\left[\begin{array}{c}
c_{n}^{\prime} \\
d_{n}^{\prime}
\end{array}\right]+f_{n}\left(\beta,\left\{c_{n}\right\},\left\{d_{n}\right\}\right), \quad n \geq 1 .
$$

We adapt the radii-polynomial technique to check that the zero-solution is locally unique. The construction of the fixed point operator and of the bounds are very similar to Section 4.2.1 and hence omited.

Let a numerical approximate solution $\bar{x} \approx 0$ be given. Then, if the computation results in a radius $r$ so that $0 \in B_{\bar{x}}(r)$, we conclude that $x=0$ is the locally unique solution and $\beta$ is simple. 
Remark 6 i) The operator $g$ is linear in $x$, therefore the radii polynomial have degree one.

ii) The introduction of the unknown $\lambda_{0}$ is technical; its purpose is to balance the number of equations with the number of unknowns. However, since the operator $\mathcal{N}$ has no generalized eigenvectors, the system $g(x)=0$ cannot have any solutions with $\lambda_{0} \neq 0$. As a result, Lemma 8 could be rephrased to say that $X_{0}=(0,0)$ is a locally unique solution of $G(X)=0$ if and only if the eigenvalue $\beta$ is simple.

\section{$5 \quad$ Numerical results}

\subsection{Checking $\Gamma$}

Suppose the Fourier coefficients $c_{n}, d_{n}$ of $w(x)$ and $z(x)$ have been proved to be in a ball of radius $r$ in the space $X^{s}$ around the numerical approximation $\bar{c}_{n}, \bar{d}_{n}$. This means that

$$
\left|c_{n}-\bar{c}_{n}\right| \leq \frac{r}{w_{n}^{s}}, \quad\left|d_{n}-\bar{d}_{n}\right| \leq \frac{r}{w_{n}^{s}}, \quad \forall n \geq 1 .
$$

We can then finally check condition (A) of the introduction; i.e. we verify that the $\Gamma$-coefficients are bounded away from zero. It can be shown [6] that $\Gamma \propto\left[\Psi^{(2)}\right]^{\prime}(1) \propto \sum_{n \geq 1}(-1)^{n} n\left(c_{n}-d_{n}\right)$ (where " $\propto$ " means "proportional"), so the enclosure of the Fourier coefficients implies

$$
\begin{aligned}
\Gamma \propto \sum_{n \geq 1}(-1)^{n} n\left(c_{n}-d_{n}\right) & \in \sum_{n=1}^{m}(-1)^{n} n\left(\bar{c}_{n}-\bar{d}_{n}\right) \pm r \sum_{n=1}^{\infty} \frac{1}{n^{s-1}}(1+i) \\
& \in \sum_{n=1}^{m}(-1)^{n} n\left(\bar{c}_{n}-\bar{d}_{n}\right) \pm r\left(1+\frac{1}{s-2}\right)(1+i),
\end{aligned}
$$

Thus, if zero does not belong to the set on the right hand side of (51), $\Gamma$ does not vanish.

\subsection{Results}

We now describe some of the computational results obtained by the method discussed above. The results are rigorous, since all computations are performed in interval arithmetics.

\subsubsection{Bounded state solution of the NLS}

Table 1 below shows the results for the ground state, as well as the first and second exited states - i.e. solutions $\phi(x)$ of (5a), (5b) with $j=0,1,2$ - for the focusing ( $\sigma=1$, left half) and defocusing $(\sigma=-1$, right half) cases and three values of the chemical potential $\mu$. The numerical solution $\bar{b}$ of the Galerkin projection $f^{m}(b)=0$ was computed by the Newton method to accuracy $\left|f^{m}(\bar{b})\right|<10^{-13}$. The table lists the finite-dimensional parameter $m_{\phi}$, the decay-rate parameter $s_{\phi}$ and the radius of the ball in the space $X^{s_{\phi}}$ around the numerical solution $\bar{b}$ within which the solution of the infinite-dimensional problem is guaranteed to exist.

\subsubsection{Enclosure of spectra and $\Gamma$-values}

For a given value of $\mu$, we considered the three different bounded states $(j=0,1,2)$ computed previously. Representative data (two values of $\mu$; one focusing, one defocusing) for the first three (non-zero) eigenvalues and the corresponding $\Gamma$-values are listed in Tables 2 and $3 . r$ denotes the radius of the ball in the space $X^{s}$ (around the approximate eigenvalue and associated eigenfunction) within which the "true" solution $\left(\beta,\left\{c_{k}, d_{k}\right\}\right)$ of $(42)$ is guaranteed to exist. The last column contains the enclosure intervals of the corresponding $\Gamma$-values. 


\begin{tabular}{ccccccccccccc}
$\sigma$ & Nodes & $\mu$ & $m_{\phi}$ & $s_{\phi}$ & $r_{\phi}$ & & $\sigma$ & Nodes & $\mu$ & $m_{\phi}$ & $s_{\phi}$ & $r_{\phi}$ \\
\hline 1 & 0 & 12.898 & 18 & 4 & $4.0089 \cdot 10^{-13}$ & & -1 & 0 & 89.237 & 20 & 4 & $9.0256 \cdot 10^{-8}$ \\
1 & 0 & 43.273 & 24 & 4 & $4.7045 \cdot 10^{-10}$ & & -1 & 0 & 161.521 & 30 & 3.5 & $3.1080 \cdot 10^{-9}$ \\
1 & 0 & 80.518 & 30 & 3.5 & $2.9894 \cdot 10^{-9}$ & & -1 & 0 & 254.916 & 36 & 3.1 & $6.7484 \cdot 10^{-9}$ \\
1 & 1 & 12.898 & 30 & 4 & $7.6398 \cdot 10^{-13}$ & & -1 & 1 & 89.237 & 20 & 4 & $4.9679 \cdot 10^{-8}$ \\
1 & 1 & 43.273 & 30 & 3.5 & $1.3889 \cdot 10^{-11}$ & & -1 & 1 & 161.521 & 30 & 3.1 & $8.4114 \cdot 10^{-10}$ \\
1 & 1 & 80.518 & 30 & 3.2 & $2.5216 \cdot 10^{-8}$ & & -1 & 1 & 254.916 & 34 & 3.1 & $4.0952 \cdot 10^{-8}$ \\
1 & 2 & 12.898 & 44 & 3.1 & $5.5127 \cdot 10^{-12}$ & & -1 & 2 & 89.237 & 16 & 4 & $2.2678 \cdot 10^{-12}$ \\
1 & 2 & 43.273 & 58 & 3.1 & $1.0442 \cdot 10^{-11}$ & & -1 & 2 & 161.521 & 54 & 3.5 & $1.2129 \cdot 10^{-11}$ \\
1 & 2 & 80.518 & 80 & 3 & $3.7820 \cdot 10^{-14}$ & -1 & 2 & 254.916 & 54 & 3 & $1.0558 \cdot 10^{-14}$
\end{tabular}

Table 1: Enclosure of bound states
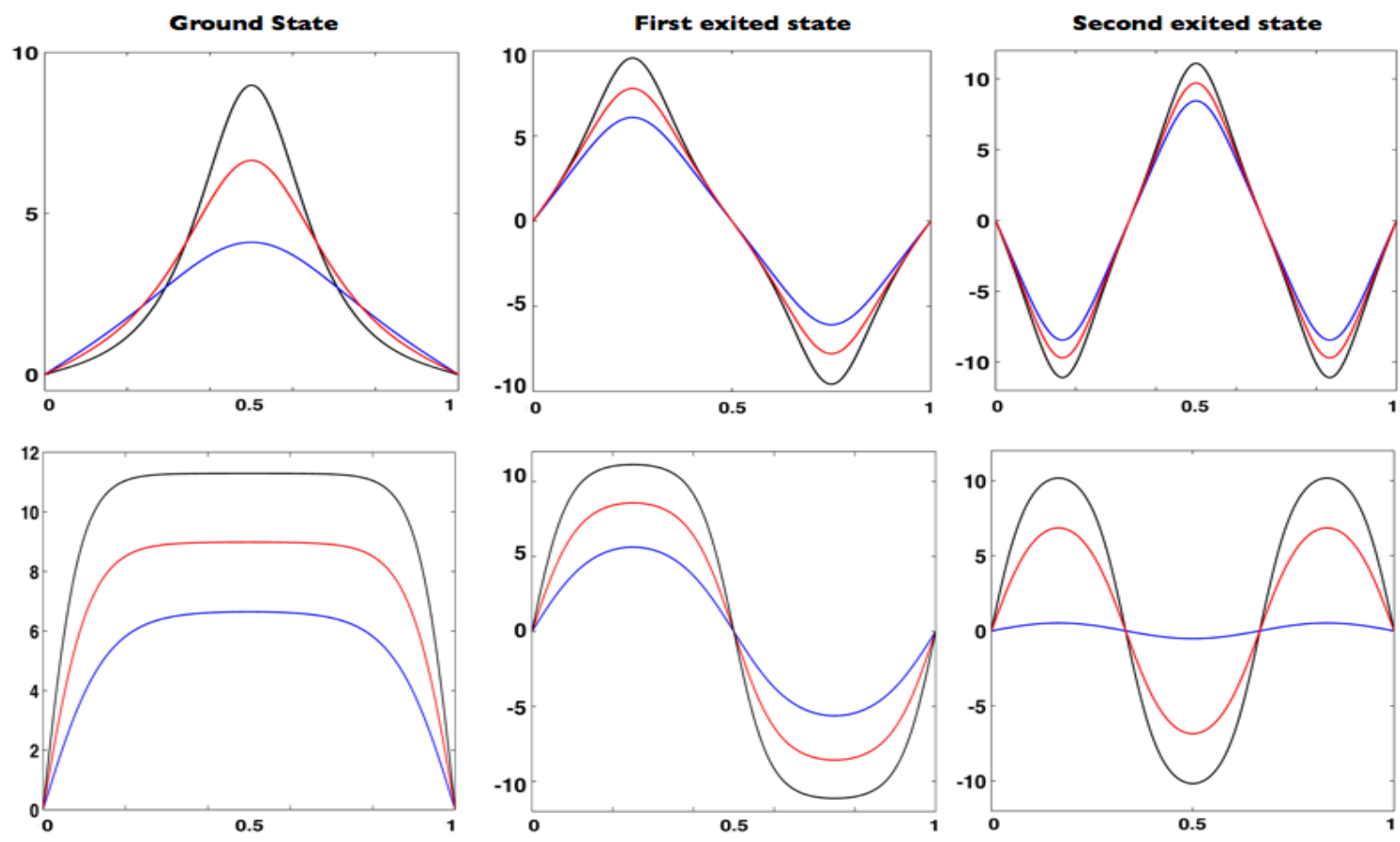

Figure 1: Bounded states for three values of $\mu$ (increasing blue $\rightarrow$ red $\rightarrow$ black). Top row: focusing case $(\sigma=1)$; bottom row: defocusing case $(\sigma=-1)$. 


\begin{tabular}{c|c|c|c|c} 
& Eigenvalue & $s$ & $r$ & $\Gamma \in$ \\
\hline \multirow{3}{*}{ Ground State } & 13.413 & 3 & $1.189 \cdot 10^{-7}$ & $0.9771 \pm 2.379 \cdot 10^{-7}$ \\
& 238.868 & 3 & $0.355 \cdot 10^{-7}$ & $-4.6741 \pm 0.709 \cdot 10^{-7}$ \\
& 791.201 & 3 & $0.622 \cdot 10^{-7}$ & $-8.8452 \pm 1.243 \cdot 10^{-7}$ \\
\hline \multirow{3}{*}{$1^{\text {st }}$ Exited State } & 90.461 & 3 & $2.027 \cdot 10^{-8}$ & $3.4138 \pm 4.0546 \cdot 10^{-8}$ \\
& 426.79 & 3 & $1.842 \cdot 10^{-8}$ & $-6.5821 \pm 3.685 \cdot 10^{-8}$ \\
& 743.05 & 3 & $2.264 \cdot 10^{-8}$ & $-8.6971 \pm 4.5283 \cdot 10^{-8}$ \\
& $40.30 \pm 15.51 i$ & 3 & $6.258 \cdot 10^{-8}$ & $(-0.4929 \pm 1.3720 i) \pm(1+i) \cdot 1.2517 \cdot 10^{-7}$ \\
\hline \multirow{3}{*}{$2^{\text {nd }}$ Exited State } & 221.73 & 3 & $1.357 \cdot 10^{-9}$ & $5.462 \pm 4.763 \cdot 10^{-9}$ \\
& 676.54 & 3 & $1.821 \cdot 10^{-9}$ & $-8.4667 \pm 5.003 \cdot 10^{-9}$ \\
& $59.95 \pm 25.55 i$ & 3 & $2.932 \cdot 10^{-9}$ & $(0.6855 \mp 1.5570 i) \pm(1+i) \cdot 5.863 \cdot 10^{-9}$ \\
& $120.36 \pm 33.13 i$ & 3 & $2.402 \cdot 10^{-9}$ & $(0.4625 \mp 2.8174 i) \pm(1+i) \cdot 4.804 \cdot 10^{-9}$
\end{tabular}

Table 2: Eigenvalues and $\Gamma s$ : focusing case, $\mu=43.273$

\begin{tabular}{c|c|c|c|c} 
& Eigenvalue & $s$ & $r$ & $\Gamma \in$ \\
\hline \multirow{3}{*}{ Ground State } & 78.671 & 3 & $5.1339 \cdot 10^{-6}$ & $1.7575 \pm 1.0268 \cdot 10^{-5}$ \\
& 360.29 & 3 & $2.0547 \cdot 10^{-6}$ & $-5.7589 \pm 4.1094 \cdot 10^{-6}$ \\
& 943.45 & 3 & $3.3776 \cdot 10^{-6}$ & $-9.8213 \pm 6.7551 \cdot 10^{-6}$ \\
\hline \multirow{3}{*}{$1^{\text {st }}$ Exited State } & 5.1026 & 3 & $2.2796 \cdot 10^{-4}$ & $3.7268 \cdot 10^{-3} \pm 4.5592 \cdot 10^{-4}$ \\
& 284.60 & 3 & $1.0601 \cdot 10^{-5}$ & $-5.1979 \pm 2.1203 \cdot 10^{-5}$ \\
& 861.30 & 3 & $9.8321 \cdot 10^{-6}$ & $-9.6062 \pm 1.9664 \cdot 10^{-5}$ \\
\hline \multirow{2}{*}{$2^{\text {nd }}$ Exited State } & 24.184 & 2.8 & $5.277 \cdot 10^{-12}$ & $-0.130 \pm 2.356 \cdot 10^{-11}$ \\
& 452.93 & 2.8 & $1.176 \cdot 10^{-12}$ & $-7.229 \pm 3.993 \cdot 10^{-12}$ \\
& 774.05 & 2.8 & $1.369 \cdot 10^{-12}$ & $-9.397 \pm 4.067 \cdot 10^{-12}$
\end{tabular}

Table 3: Eigenvalues and $\Gamma s$ : defocusing case, $\mu=254.916$
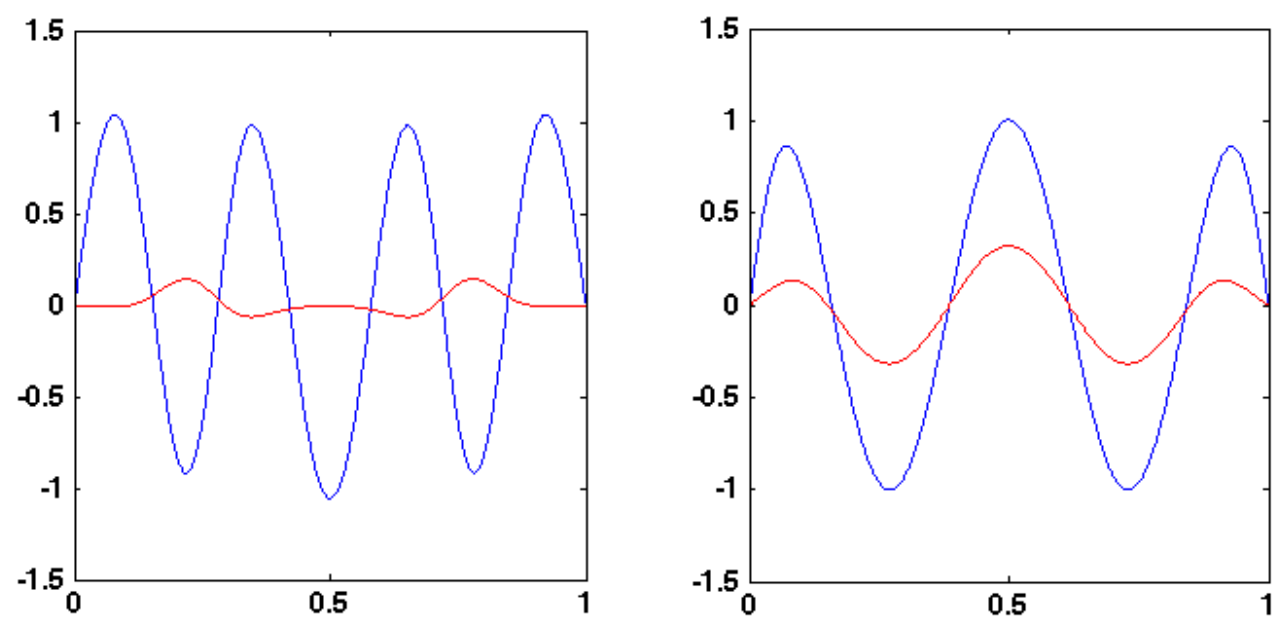

Figure 2: Eigenfunctions $w(x)$ (blue) and $z(x)$ (red); cf. equations $(19 \mathrm{c}),(19 \mathrm{~d})$. Left panel: eigenfunction associated with eigenvalue $\beta=743.05$ for the first exited state with $\sigma=1$ (focusing). Right panel: $\beta=360.29$ for the ground state and $\sigma=-1$ (defocusing). 
Remark 7 It may be surprising and/or confusing that the eigenvalues and $\Gamma$ values as well as their enclosure intervals are sometimes written in real form and sometimes written in complex form. To explain this, we first note that all computations are carried out in complex Banach spaces. However, if the numerical (approximate) solution (i.e the centre of the enclosure interval) is real, then the exact solution is real as well. Indeed, if the (exact) solution was complex, its complex conjugate would be a solution as well, which would fall in the same enclosure ball. This is impossible by uniqueness.

\section{Concluding remarks}

In this paper we analyzed important aspects of a realistic model for a one-dimensional BEC by numerical means. Since the results are derived from a computational scheme that is based on the radii-polynomial technique in conjunction with interval arithmetic, they are mathematically rigorous and can be used to complement and complete analytical proofs, such as the controllability proof given in [6]. The method adopted is general and flexible; as a result, both the focusing and defocusing cases as well as ground and excited states can be treated within the same computational framework. Specifically, we

(i) rigorously computed the ground and (the first two ${ }^{3}$ excited states;

(ii) rigorously computed finitely many eigenvalues and eigenfunctions of the linearization (around the bound states determined previously);

(iii) proved (by rigorous numerics) that the eigenvalues are simple (B);

(iv) rigorously verified the " $\Gamma$-condition" (A).

The model studied in this paper has considerable interest in its stated form, both from the physical and the mathematical point of view (as for the latter, we note that only very few applications of rigorous numerics to infinite-dimensional problems exist to date). However, there are some obvious generalizations that immediately come to mind, such as the whole-space problem (with a suitable potential, such as the harmonic oscillator) to replace Dirichlet boundary conditions and/or higher space dimensions. These generalizations are subject to current research by the authors and will be reported on in the future.

Furthermore, in addition to presenting a study of an (important) particular model, we also view this paper as a case study that illustrates the general utility and flexibility of the rigorous-numerics paradigm. We believe that the latter will find applications with other important problems in mathematics and science and will thus become a valuable tool in the arsenal of mathematicians, physicists, and scientists at large.

\section{Acknowledgements}

This work was partially supported by grant MTM2011-24766 of the MICINN, Spain, and the Natural Sciences and Engineering Research Council of Canada (NSERC). This research was begun during a three-month stay of the second author at the Basque Center for Applied Mathematics (BCAM), who would like to thank BCAM for its hospitality and financial support.

\footnotetext{
${ }^{3}$ This number is completely arbitrary; there is no restriction in principle to rigorously determining any number of bound states. A similar comment applies to the number of eigenvalues of the linearization.
} 


\section{Appendix}

The integral

$(52)$

$$
\begin{gathered}
\int_{0}^{1} \sin (\pi k x) \sin (\pi l x) \sin (\pi p x) \sin (\pi n x) d x= \\
\frac{1}{8}\left(\delta_{p+k-l-n}-\delta_{p+k-l+n}+\delta_{p-k+l-n}-\delta_{p-k+l+n}-\delta_{p+k+l-n}+\delta_{p+k+l+n}-\delta_{p-k-l-n}+\delta_{p-k-l+n}\right)
\end{gathered}
$$

is readily computed. Hence, given $\phi(x)=\sum_{n \in \mathbb{Z}} b_{n} \sin (n \pi x)$, we have

$$
\begin{aligned}
<\phi^{3}, \sqrt{2} \sin (\pi n \bullet)> & =4 \int_{0}^{1}\left[\sum_{k \in \mathbb{Z}} b_{k} \sin (\pi k x) \sum_{\ell \in \mathbb{Z}} b_{\ell} \sin (\pi l x) \sum_{p \in \mathbb{Z}} b_{p} \sin (\pi p x)\right] \sin (\pi n x) d x \\
& =4 \sum_{k, \ell, p \in \mathbb{Z}} b_{k} b_{\ell} b_{p} \int_{0}^{1} \sin (\pi k x) \sin (\pi l x) \sin (\pi p x) \sin (\pi n x) d x .
\end{aligned}
$$

Using (52) and the property $b_{-k}=-b_{k}$, eq. (37) follows.

\subsection{Appendix A: analytical estimates for the enclosure of $\phi(x)$}

\section{Bounds}

The definition of the bounds $Y, Z$ is the same as in [18], so we refer to that paper for a detailed explanation. We first recall the definition of some constants:

$$
\begin{aligned}
& \gamma_{k}=2\left[\frac{k}{k-1}\right]^{s}+\left[\frac{4 \ln (k-2)}{k}+\frac{\pi^{2}-6}{3}\right]\left[\frac{2}{k}+\frac{1}{2}\right]^{s-2} \\
& \alpha_{k}^{(2)}= \begin{cases}4+\frac{1}{2^{2 s-1}(2 s-1)} & k=0 \\
2\left[2+\frac{1}{2^{s}}+\frac{1}{3^{s}}+\frac{1}{3^{s-1}(s-1)}\right]+\sum_{k_{1}=1}^{k-1} \frac{k^{s}}{k_{1}^{s}(k-k 1)^{s}} & 1 \leq k \leq M-1 \\
2\left[2+\frac{1}{2^{s}}+\frac{1}{3^{s}}+\frac{1}{3^{s-1}(s-1)}\right]+\gamma_{k} & k \geq M\end{cases} \\
& \alpha_{k}^{(3)}= \begin{cases}\alpha_{0}^{(2)}+2 \sum_{k_{1}=1}^{M-1} \frac{\alpha_{k_{1}}^{(2)}}{k_{1}^{2 s}}+\frac{2 \alpha_{M}^{(2)}}{(M-1)^{2 s-1}(2 s-1)} & k=0 \\
\sum_{k_{1}=1}^{M-1} \frac{\alpha_{k_{1}+k^{s}} k^{s}}{k_{1}^{s}\left(k+k_{1}\right)^{s}}+\alpha_{M}^{(2)} k^{s}\left[\frac{1}{(M-k)^{s} M^{s}}+\frac{1}{(M-k)^{s-1} M^{s}(s-1)}\right] & \\
+\alpha_{k}^{(2)}+\sum_{k_{1}=1}^{k-1} \frac{\alpha_{k_{1}} k^{s}}{k_{1}^{s}\left(k-k_{1}\right)^{s}}+\alpha_{0}^{(2)}+\sum_{k_{1}=1}^{M-1} \frac{\alpha_{k_{1}}^{(2)} k^{s}}{k_{1}^{s}\left(k+k_{1}\right)^{s}}+\frac{\alpha_{M}^{(2)}}{(M-1)^{s-1}(s-1)} & 1 \leq k \leq M-1 \\
\alpha_{M}^{(2)}\left[2+\frac{1}{2^{s}}+\frac{1}{3^{s}}+\frac{1}{3^{s-1}(s-1)+\frac{1}{(M-1)^{s-1}(s-1)}}+\gamma_{k}\right] & \\
+\alpha_{0}^{(2)}+\sum_{k_{1}=1}^{M-1}\left(\frac{\alpha_{k_{1}}^{(2)}}{k_{1}^{s}}\left[1+\frac{M^{s}}{\left(M-k_{1}\right)^{s}}\right]\right) & k \geq M\end{cases} \\
& \varepsilon_{k}^{(3)}=\frac{2 \alpha_{M}^{(2)}}{(s-1)(M-1)^{s-1}(M+k)^{s}}+\sum_{k_{1}=M}^{M+k-1} \frac{\alpha_{k_{1}-k}^{(2)}}{w_{k_{1}}^{s} w_{k_{1}-k}^{s}} \\
& \tilde{\alpha}_{M}^{(3)}:=\max \left\{\alpha_{k}^{(3)}: k=0 \ldots M\right\} .
\end{aligned}
$$

Define the bound $Y$ as

$$
Y_{k}:=\left\{\begin{array}{ll}
\left|\left[A^{(m)} f^{m}(\bar{b})\right]_{k}\right|, & k=1, \ldots, m \\
\left|\Lambda_{k}^{-1} f_{k}(\bar{b})\right| & k=m+1, \ldots, M-1 \\
0 & k \geq M
\end{array} .\right.
$$


For the bound $Z_{k}$ we first define

$$
\left(Z^{0}\right)_{k}=\left\{\begin{array}{ll}
{\left[\left|I-A^{m} D f^{(m)}\right|\left\{w_{j}^{-s}\right\}_{j=1}^{m}\right]_{k},} & k=1, \ldots, m \\
0, & k>m
\end{array} .\right.
$$

and

$$
\left(Z^{1}\right)_{k}=\left\{\begin{array}{cc}
\sum_{\substack{k_{1}+k_{2}+k_{3}=k \\
\left|k_{1}\right|,\left|k_{2}\right|<m, m \leq\left|k_{3}\right|<M}}\left|\bar{b}_{k_{1}}\right|\left|\bar{b}_{k_{2}}\right| \frac{1}{w_{k_{3}}^{s}}+\|\bar{b}\|_{s}^{2} \varepsilon_{k}^{(3)} & k=1, \ldots, m \\
\sum_{\substack{k_{1}+k_{2}+k_{3}=k \\
\left|k_{1}\right|,\left|k_{2}\right|<m,\left|k_{3}\right|<M}}\left|\bar{b}_{k_{1}}\right|\left|\bar{b}_{k_{2}}\right| \frac{1}{w_{k_{3}}^{s}}+\|\bar{b}\|_{s}^{2} \varepsilon_{k}^{(3)} & m+1 \leq k<M
\end{array} .\right.
$$

and, for any $1 \leq k<M$,

$$
\begin{gathered}
\left(Z^{2}\right)_{k}:=\sum_{\substack{k_{1}+k_{2}+k_{3}=k \\
\left|k_{1}\right|<m,\left|k_{2}\right|,\left|k_{3}\right|<M}}\left|\bar{b}_{k_{1}}\right| \frac{1}{w_{k_{2}}^{s}} \frac{1}{w_{k_{3}}^{s}}+2\|\bar{b}\|_{s} \varepsilon_{k}^{(3)} \\
\left(Z^{3}\right)_{k}:=\sum_{\substack{k_{1}+k_{2}+k_{3}=k \\
\left|k_{j}\right|<M}} \frac{1}{w_{k_{3}}^{s}} \frac{1}{w_{k_{2}}^{s}} \frac{1}{w_{k_{3}}^{s}}+3 \varepsilon_{k}^{(3)} .
\end{gathered}
$$

Collecting all the terms, we have

$$
Z_{k}:=\left\{\begin{array}{ll}
6\left[\left|A^{m}\right|\left(\left(Z^{1}\right)^{m} r+2\left(Z^{2}\right)^{m} r^{2}+\left(Z^{3}\right)^{m} r^{3}\right]_{k}+Z_{k}^{0} r\right. & 1 \leq k<m \\
6\left|\mu_{k}^{-1}\right|\left(Z_{k}^{1} r+2 Z_{k}^{2} r^{2}+Z_{k}^{3} r^{3}\right) & m \leq k<M
\end{array} .\right.
$$

The tail bound $Y_{M}$ can be set equal to zero, while

$$
Z_{M}=6 C_{\Lambda}\left(\|\bar{b}\|_{s}^{2} \tilde{\alpha}_{M}^{(3)} r+2\|\bar{b}\|_{s} \tilde{\alpha}_{M}^{(3)} r^{2}+\tilde{\alpha}_{M}^{(3)} r^{3}\right) .
$$

Finally, the radii polynomials are

$$
\begin{gathered}
p_{k}(r)=Y_{k}+\left(Z_{k}^{0}+6\left[\left|A^{(m)}\right|\left(Z^{1}\right)^{m}\right]_{k}-1 / w_{k}^{s}\right) r+\left(12\left[\left|A^{(m)}\right|\left(Z^{2}\right)^{m}\right]_{k}\right) r^{2}+\left(6\left[\left|A^{(m)}\right|\left(Z^{3}\right)^{m}\right]_{k}\right) r^{3}, \quad 1 \leq k \leq m \\
p_{k}(r)=Y_{k}+\left(\frac{6 Z_{k}^{1}}{\left|\mu_{k}\right|}-\frac{1}{w_{k}^{s}}\right) r+\frac{12 Z_{k}^{2}}{\left|\mu_{k}\right|} r^{2}+\frac{6 Z_{k}^{3}}{\left|\mu_{k}\right|} r^{3}, \quad m<k<M . \\
p_{M}(r)=6 C_{\Lambda} \tilde{\alpha}_{M}^{(3)} r^{2}+12 C_{\Lambda}\|\bar{\beta}\|_{s} \tilde{\alpha}_{M}^{(3)} r+6 C_{\Lambda}\|\bar{\beta}\|_{s}^{2} \tilde{\alpha}_{M}^{(3)}-1
\end{gathered}
$$

\subsection{Appendix B}

\section{Construction of the matrix $F$}

The matrix $F=\left\{F_{n, \ell}\right\}$ is defined by

$$
<\phi^{2} y, \sqrt{2} \sin (\pi n \bullet)>=\sum_{\ell \geq 1} F_{n, \ell} \xi_{\ell}
$$


where $y=\sqrt{2} \sum_{k \geq 1} \xi_{k} \sin (\pi k x)$ and $\phi(x)=\sqrt{2} \sum_{k \geq 1} \alpha_{k} \sin (\pi k x)$. Now, by 52 and using the symmetry $b_{-k}=-\bar{b}_{k}$,

$$
\begin{gathered}
<\phi^{2} y, \sqrt{2} \sin (\pi n \bullet)>= \\
4 \sum_{p, k \in \mathbb{Z}, \ell \geq 1} \alpha_{p} \alpha_{k} \xi_{\ell} \int_{0}^{1} \sin (\pi k x) \sin (\pi \ell x) \sin (\pi p x) \sin (\pi n x) d x= \\
\frac{1}{2} \sum_{\ell \geq 1}\left[\sum_{p+k=\ell+n} b_{p} b_{k}-\sum_{p+k=\ell-n} b_{p} b_{k}+2 \sum_{p-k=n-\ell} b_{p} b_{k}-2 \sum_{p-k=\ell+n} b_{p} b_{k}-\sum_{p+k=n-\ell} b_{p} b_{k}+\sum_{p+k=-n-\ell} b_{p} b_{k}\right]= \\
\frac{1}{2} \sum_{\ell \geq 1} \xi_{\ell}\left[4 \sum_{p+k=n+\ell} b_{p} b_{k}-4 \sum_{p+k=\ell-n} b_{p} b_{k}\right]
\end{gathered}
$$

giving

$$
F_{n, \ell}=2\left[\sum_{p+k=n+\ell} b_{p} b_{k}-\sum_{p+k=\ell-n} b_{p} b_{k}\right]
$$

Bound for $\left\|\Lambda_{k}^{-1}\right\|_{\infty}$

Recall the definition of $\Lambda_{k}$ :

$$
\Lambda_{k}=\frac{\partial F_{k}}{\partial\left(c_{k}, d_{k}\right)}(\bar{x})=\left[\begin{array}{cc}
\pi^{2} k^{2}+\sigma \mu-\bar{\beta}-2 \sigma F_{k, k} & \sigma F_{k, k} \\
\sigma F_{k, k} & \pi^{2} k^{2}+\sigma \mu+\bar{\beta}-2 \sigma F_{k, k}
\end{array}\right] .
$$

Since these are diagonally dominated matrices, we have that

$$
\left\|\Lambda^{-1}\right\|_{\infty} \leq \max \left\{\frac{1}{\left|\pi^{2} k^{2}+\sigma \mu-\bar{\beta}-2 \sigma F_{k, k}\right|-\left|F_{k, k}\right|}, \frac{1}{\left|\pi^{2} k^{2}+\sigma \mu+\bar{\beta}-2 \sigma F_{k, k}\right|-\left|F_{k, k}\right|}\right\} .
$$

If $k$ is large enough, both denominators are greater than

$$
\pi^{2} k^{2}+\sigma \mu-|\bar{\beta}|-3\left|F_{k, k}\right|=k^{2}\left(\pi^{2}+\frac{\sigma \mu}{k^{2}}-\frac{1}{k^{2}}\left(|\bar{\beta}|+3\left|F_{k, k}\right|\right)\right) .
$$

For $k>m$ and assuming $m>2 m_{\phi}$,

$$
\left|F_{k, k}\right| \leq 2\left|\sum_{p_{1}+p_{2}=0} \bar{b}_{p_{1}} \bar{b}_{p_{2}}\right|+2 \mathcal{E}(2 k)+2 \mathcal{E}(0) \leq 2\left|\sum_{p_{1}+p_{2}=0} \bar{b}_{p_{1}} \bar{b}_{p_{2}}\right|+2 \mathcal{E}(2 m)+2 \mathcal{E}(0)=: C_{F} .
$$

Therefore for any $k>m$

$$
\left\|\Lambda^{-1}\right\|_{\infty} \leq \frac{\mathcal{C}_{\Lambda}(m)}{k^{2}}
$$

with

$$
\mathcal{C}_{\Lambda}(m):=\frac{1}{\pi^{2}+\frac{\sigma \mu}{(m+1)^{2}}-\frac{1}{(m+1)^{2}}\left(|\bar{\beta}|+3 C_{F}\right)}
$$

Proof of Lemma 3.

In view of 41) we have

$$
\begin{aligned}
\sum_{p+k=q} b_{p} b_{k} & \in \sum_{p+k=q}\left(\bar{b}_{p} \pm \frac{r_{\phi} 2^{s_{\phi}}}{w_{p}^{s_{\phi}}}\right)\left(\bar{b}_{k} \pm \frac{r_{\phi} 2^{s_{\phi}}}{w_{k}^{s_{\phi}}}\right) \\
& \in \sum_{p+k=q} \bar{b}_{p} \bar{b}_{k} \pm\left(2 r_{\phi} 2^{s_{\phi}} \sum_{p+k=q}\left|\bar{b}_{p}\right| w_{k}^{-s_{\phi}}+r_{\phi}^{2} 4^{s_{\phi}} \sum_{p+k=q} w_{k}^{-s_{\phi}} w_{p}^{-s_{\phi}}\right)
\end{aligned}
$$


Then

$$
\left|\sum_{p+k=q} b_{p} b_{k}\right| \leq\left|\sum_{p+k=q} \bar{b}_{p} \bar{b}_{k}\right|+\left(2 r_{\phi} 2^{s_{\phi}} \sum_{p+k=q}\left|\bar{b}_{p}\right| w_{k}^{-s_{\phi}}+r_{\phi}^{2} 4^{s_{\phi}} \sum_{p+k=q} w_{k}^{-s_{\phi}} w_{p}^{-s_{\phi}}\right) .
$$

Using

$$
\sum_{k_{1}+k_{2}=q} \frac{1}{w_{k_{1}}^{s_{\phi}}} \frac{1}{w_{k_{2}}^{s_{\phi}}} \leq \frac{\alpha_{q}^{(2)}}{w_{q}^{s_{\phi}}}
$$

[18, Lemma A.3], gives the first assertion. Moreover, since $\bar{b}_{k}=0$ for $|k|>2 m_{\phi}$, the first sum is equal to zero whenever $|q|>4 m_{\phi}$.

\section{Proof of Lemma 5 .}

Since $\bar{x}_{k}=0$ for $k>m$, we have

$$
f_{k}(\bar{x})=\left[\begin{array}{r}
-\sum_{1 \leq \ell \leq m} F_{k, \ell}\left(2 \bar{c}_{\ell}-\bar{d}_{\ell}\right) \\
\sum_{1 \leq \ell \leq m} F_{k, \ell}\left(\bar{c}_{\ell}-2 \bar{d}_{\ell}\right)
\end{array}\right], \quad \forall k>m
$$

and so

$$
\left|f_{k}(\bar{x})\right| \leq \sum_{1 \leq \ell \leq m}\left|F_{k, \ell}\right|\left[\begin{array}{c}
\left|\left(2 \bar{c}_{\ell}-\bar{d}_{\ell}\right)\right| \\
\left.\mid \bar{c}_{\ell}-2 \bar{d}_{\ell}\right) \mid
\end{array}\right], \quad \forall k>m .
$$

If $k \geq M$ we have that $k-m \geq 4 m_{\phi}$; hence, by 49,

$$
\begin{aligned}
\left|f_{k}(\bar{x})\right| & \leq 2 \sum_{1 \leq \ell \leq m}\left(\frac{1}{(k+\ell)^{s_{\phi}}} \tilde{\mathcal{E}}(k+\ell)+\frac{1}{(k-\ell)^{s_{\phi}}} \tilde{\mathcal{E}}(k-\ell)\right)\left[\begin{array}{r}
\left|\left(2 \bar{c}_{\ell}-\bar{d}_{\ell}\right)\right| \\
\left.\mid \bar{c}_{\ell}-2 \bar{d}_{\ell}\right) \mid
\end{array}\right] \\
& \leq \frac{2}{k^{s_{\phi}}} \sum_{1 \leq \ell \leq m}\left(\frac{1}{\left(1+\frac{l}{k}\right)^{s_{\phi}}} \tilde{\mathcal{E}}(k+\ell)+\frac{1}{\left(1-\frac{l}{k}\right)^{s_{\phi}}} \tilde{\mathcal{E}}(k-\ell)\right)\left[\begin{array}{r}
\left|\left(2 \bar{c}_{\ell}-\bar{d}_{\ell}\right)\right| \\
\left.\mid \bar{c}_{\ell}-2 \bar{d}_{\ell}\right) \mid
\end{array}\right] \quad \forall k \geq M .
\end{aligned}
$$

From the monotonicity of $\tilde{\mathcal{E}}(k)$ it follows that for any $k \geq M$

$$
\left|f_{k}(\bar{x})\right| \leq \frac{2}{k^{s_{\phi}}} \sum_{1 \leq \ell \leq m}\left(\tilde{\mathcal{E}}(M+\ell)+\frac{1}{\left(1-\frac{l}{M}\right)^{s_{\phi}}} \tilde{\mathcal{E}}(M-\ell)\right)\left[\begin{array}{c}
\left|\left(2 \bar{c}_{\ell}-\bar{d}_{\ell}\right)\right| \\
\left.\mid \bar{c}_{\ell}-2 \bar{d}_{\ell}\right) \mid
\end{array}\right]=: \frac{1}{k^{s_{\phi}}} \mathcal{H}(M) .
$$

\section{Proof of Lemma 6.}

For any $k=1, \ldots, m$

$$
\begin{aligned}
\sum_{j=m+1}^{\infty}\left|F_{k, j}\right| j^{-s} \leq & 2 \sum_{j=m+1}^{4 m_{\phi}+k}\left(\left|\sum_{p_{1}+p_{2}=k+j} \bar{p}_{1} \bar{p}_{2}\right|+\left|\sum_{p_{1}+p_{2}=j-k} \bar{p}_{1} \bar{p}_{2}\right|\right) j^{-s} \\
& +2 \sum_{j=m+1}^{\infty}(\mathcal{E}(k+j)+\mathcal{E}(j-k)) j^{-s}
\end{aligned}
$$

Moreover,

$$
\begin{aligned}
\sum_{j=m+1}^{\infty}\left|F_{k, j}\right| j^{-s} & \leq \sum_{j=m+1}^{4 m_{\phi}+k}\left|\bar{F}_{k, j}\right| j^{-s}+2 \sum_{j=m+1}(\mathcal{E}(m+1+k)+\mathcal{E}(m+1-k)) j^{-s} \\
& \leq \sum_{j=m+1}^{4 m_{\phi}+k}\left|\bar{F}_{k, j}\right| j^{-s}+2 \frac{\mathcal{E}(m+1+k)+\mathcal{E}(m+1-k)}{(s-1) m^{s-1}}
\end{aligned}
$$


For $k>m$

$$
\begin{aligned}
\sum_{j=1, j \neq k}^{\infty}\left|F_{k, j}\right| j^{-s} \leq & \sum_{j=\max \left\{1, k-4 m_{\phi}\right\}, j \neq k}^{k+4 m_{\phi}}\left|\bar{F}_{k, j}\right| j^{-s}+2 \sum_{j=1, j \neq k}^{\infty}(\mathcal{E}(j+k)+\mathcal{E}(|j-k|)) j^{-s} \\
\leq & \sum_{j=\max \left\{1, k-4 m_{\phi}\right\}, j \neq k}^{k+4 m_{\phi}}\left|\bar{F}_{k, j}\right| j^{-s}+2 \sum_{j=1}^{k-1} \mathcal{E}(|j-k|) j^{-s} \\
& +2 \sum_{j=k+1}^{\infty} \mathcal{E}(|j-k|) j^{-s}+2 \sum_{j=1}^{\infty} \mathcal{E}(j+k) j^{-s} \\
\leq & \sum_{j=\max \left\{1, k-4 m_{\phi}\right\}, j \neq k}^{k+4 m_{\phi}}\left|\bar{F}_{k, j}\right| j^{-s}+2 \sum_{j=1}^{k-1} \mathcal{E}(k-j \mid) j^{-s} \\
+ & \mathcal{E}(1) \frac{2}{(k+1)^{s}}+\mathcal{E}(2) \frac{2}{(s-1)(k+1)^{s-1}}+\mathcal{E}(k+1)+\mathcal{E}(k+2) \frac{2}{(s-1)}
\end{aligned}
$$

Proof of Lemma 7

We need to find $Z_{M}^{1}$ such that

$$
\left|c_{k, 1}\right|_{\infty} \leq \frac{1}{w_{k}^{s}} Z_{M}^{1} \quad k \geq M
$$

This requires a uniform bound for $\left|c_{k, 1}\right|_{\infty}$ as $k \geq M$. First we have

$$
\sum_{j=1, j \neq k}^{\infty}\left|F_{k, j}\right| j^{-s} \leq \sum_{j=k-4 m_{\phi}, j \neq k}^{k+4 m_{\phi}}\left|\bar{F}_{k, j}\right| j^{-s}+2 \sum_{j=1, j \neq k}^{\infty}(\mathcal{E}(j+k)+\mathcal{E}(|j-k|)) j^{-s}
$$

Since $M>m+4 m_{\phi}$,

$$
\begin{aligned}
\sum_{j=k-4 m_{\phi}, j \neq k}^{k+4 m_{\phi}}\left|\bar{F}_{k, j}\right| j^{-s} & =2 \sum_{p=-4 m_{\phi}, p \neq 0}^{4 m_{\phi}}\left|\sum_{p_{1}+p_{2}=p} \bar{b}_{p_{1}} \bar{b}_{p_{2}}\right| \cdot(k-p)^{-s} \\
& =\frac{2}{k^{s}} \sum_{p=1}^{4 m_{\phi}}\left|\sum_{p_{1}+p_{2}=p} \bar{b}_{p_{1}} \bar{b}_{p_{2}}\right| \cdot \frac{1}{\left(1-\frac{p}{k}\right)^{s}+\left(1+\frac{p}{k}\right)^{s}} \\
& \leq \frac{2}{k^{s}} \sum_{p=1}^{4 m_{\phi}}\left|\sum_{p_{1}+p_{2}=p} \bar{b}_{p_{1}} \bar{b}_{p_{2}}\right| \cdot \frac{1}{\left(1-\frac{p}{M}\right)^{s}+1}, \quad \forall k \geq M .
\end{aligned}
$$

For the remaining series in the right hand side of 70$)$, we write

$$
\begin{aligned}
\sum_{j=1, j \neq k}^{\infty}(\mathcal{E}(j+k)+\mathcal{E}(|j-k|)) j^{-s} & \leq \sum_{j=1}^{\infty} \mathcal{E}(j+k) j^{-s}+\sum_{j=1}^{k-1} \mathcal{E}(k-j) j^{-s}+\sum_{j=k+1}^{\infty} \mathcal{E}(j-k) j^{-s} \\
& \leq \sum_{j=1}^{\infty} \frac{\tilde{\mathcal{E}}(j+k)}{(j+k)^{s_{\phi}} j^{s}}+\sum_{j=1}^{k-1} \frac{\tilde{\mathcal{E}}(k-j)}{(k-j)^{s_{\phi}} j^{s}}+\sum_{j=k+1}^{\infty} \frac{\tilde{\mathcal{E}}(j-k)}{(j-k)^{s_{\phi}} j^{s}}
\end{aligned}
$$

Since $s<s_{\phi}$,

$$
\begin{aligned}
\sum_{j=1, j \neq k}^{\infty}(\mathcal{E}(j+k)+\mathcal{E}(|j-k|)) j^{-s} & \leq \frac{1}{k^{s}} \sum_{j=1}^{\infty} \frac{k^{s} \tilde{\mathcal{E}}(k+j)}{(j+k)^{s} j^{s}}+\frac{\tilde{\mathcal{E}}(1)}{k^{s}} \sum_{j=1}^{k-1} \frac{k^{s}}{(k-j)^{s} j^{s}}+\frac{1}{k^{s}} \sum_{j=k+1}^{\infty} \frac{k^{s} \tilde{\mathcal{E}}(j-k)}{(j-k)^{s} j^{s}} \\
& \leq \frac{1}{k^{s}}\left[\sum_{j=1}^{\infty} \frac{\tilde{\mathcal{E}}(k+j)}{\left(1+\frac{j}{k}\right)^{s} j^{s}}+\tilde{\mathcal{E}}(1) \gamma_{k}+\sum_{j=1}^{\infty} \frac{\tilde{\mathcal{E}}(j)}{\left(1+\frac{j}{k}\right)^{s} j^{s}}\right]
\end{aligned}
$$


where $\gamma_{k}$ is given in (54); see [18].

Therefore, for any $k \geq M$,

$$
\begin{aligned}
\sum_{j=1, j \neq k}^{\infty}(\mathcal{E}(j+k)+\mathcal{E}(|j-k|)) j^{-s} & \leq \frac{1}{k^{s}}\left[\sum_{j=1}^{\infty} \frac{\tilde{\mathcal{E}}(M+j)}{j^{s}}+\tilde{\mathcal{E}}(1) \gamma_{M}+\sum_{j=1}^{\infty} \frac{\tilde{\mathcal{E}}(j)}{j^{s}}\right] \\
& \leq \frac{1}{k^{s}}\left[\tilde{\mathcal{E}}(M+1)+\frac{\tilde{\mathcal{E}}(M+2)}{s-1}+\tilde{\mathcal{E}}(1) \gamma_{M}+\tilde{\mathcal{E}}(1)+\frac{\tilde{\mathcal{E}}(2)}{s-1}\right]
\end{aligned}
$$

Since $\left|c_{k, 1}\right|_{\infty} \leq 3 \sum_{j=1, j \neq k}^{\infty}\left|F_{k, j}\right| j^{-s}$, combining (70) with (71) and 274, the thesis follows.

\section{References}

[1] M. Abramowitz and I.A. Stegun. Handbook of mathematical functions. Dover, 1965.

[2] Gianni Arioli and Hans Koch. Computer-assisted methods for the study of stationary solutions in dissipative systems, applied to the Kuramoto-Sivashinski equation. Arch. Ration. Mech. Anal., 197(3):1033-1051, 2010.

[3] R. Asad and G. Simpson. Embedded eigenvalues and the nonlinear Schrd̈inger equation. Journal of Mathematical Physics, 52(3), 2011.

[4] Y. Band, B. Malomed, and M. Trippenbach. Adiabaticity in nonlinear quantum dynamics: Bose-Einstein condensate in a time-varying box. Phys. Rev. A, 65:033607, 2002.

[5] K. Beauchard. Controllability of a quantum particle in a 1d variable domain. ESAIM Contr. Opt. Calc. Var., 14:105-147, 2008.

[6] K. Beauchard, H. Lange, and H. Teismann. Local controllability of a Bose-Einstein condensate in a 1D time-varying box. Under review, 2013.

[7] C. Brif, R. Chakrabarti, and H. Rabitz. Control of quantum phenomena. Advances in Chemical Physics, pages 1-76, 2012.

[8] L.D. Carr, C.W. Clark, and W.P. Reinhardt. Stationary solutions of the one-dimensional nonlinear Schrödinger equation. I. Case of repulsive nonlinearity. Phys. Rev. A, 62(6):063610, 2000.

[9] L.D. Carr, C.W. Clark, and W.P. Reinhardt. Stationary solutions of the one-dimensional nonlinear Schrödinger equation. II. Case of attractive nonlinearity. Phys. Rev. A, 62:063611-21, 2000.

[10] R. Castelli and J. Lessard. Rigorous numerics in floquet theory: Computing stable and unstable bundles of periodic orbits. SIAM Journal on Applied Dynamical Systems, 12(1):204-245, 2013.

[11] Roberto Castelli and Jean-Philippe Lessard. A method to rigorously enclose eigendepairs of complex interval matrices. In Conference Applications of Mathematics 2013, in honor of the 70th birthday of Karel Segeth. Institute of Mathematics AS CR, Prague., 2013.

[12] Sarah Day, Jean-Philippe Lessard, and Konstantin Mischaikow. Validated continuation for equilibria of PDEs. SIAM J. Numer. Anal., 45(4):1398-1424 (electronic), 2007.

[13] A. del Campo and MG Boshier. Shortcuts to adiabaticity in a time-dependent box. Scientific Reports, 2, 2012. 
[14] L. Demanet and W. Schlag. Numerical verification of a gap condition for a linearized nonlinear schrödinger equation. Nonlinearity, 19(4):829, 2006.

[15] T. Dohnal, K. Nagatou, M. Plum, and W. Reichel. Interfaces supporting surface gap soliton ground states in the 1d nonlinear schroedinger equation. arXiv preprint arXiv:1202.3588, 2012.

[16] C.L. Fefferman and L.A. Seco. Interval arithmetic on quantum mechanics. In Applications of interval computations (El Paso, TX, 1995), 145167, Appl. Optim., 3.

[17] Zbigniew Galias and Piotr Zgliczyński. Infinite-dimensional Krawczyk operator for finding periodic orbits of discrete dynamical systems. Internat. J. Bifur. Chaos Appl. Sci. Engrg., 17(12):4261-4272, 2007.

[18] Marcio Gameiro and Jean-Philippe Lessard. Analytic estimates and rigorous continuation for equilibria of higher-dimensional PDEs. J. Differential Equations, 249(9):2237-2268, 2010.

[19] R. Krawczyk. Newton-Algorithmen zur Bestimmung von Nullstellen mit Fehlerschranken. Computing (Arch. Elektron. Rechnen), 4:187-201, 1969.

[20] J.L. Marzuola and G. Simpson. Spectral analysis for matrix Hamiltonian operators. Nonlinearity, 24:389-429, 2011.

[21] TP Meyrath, Schreck F., JL Hanssen, C.S. Chuu, and MG Raizen. Bose-einstein condensate in a box. Physical review. A, 71(4):041604-1, 2005.

[22] K. Nagatou, M. Plum, and M.T. Nakao. Eigenvalue excluding for perturbed-periodic onedimensional schrödinger operators. Proceedings of the Royal Society A: Mathematical, Physical and Engineering Science, 468(2138):545-562, 2012.

[23] Kaori Nagatou, Michael Plum, and Mitsuhiro T. Nakao. Eigenvalue excluding for perturbedperiodic one-dimensional schrödinger operators. Proceedings of the Royal Society A: Mathematical, Physical and Engineering Science, 102011.

[24] I. Rodnianski, W. Schlag, and A. Soffer. Asymptotic stability of N-soliton states of NLS. arXiv:math/0309114, 2005.

[25] S.M. Rump. INTLAB - INTerval LABoratory. In Tibor Csendes, editor, Developments in Reliable Computing, pages 77-104. Kluwer Academic Publishers, Dordrecht, 1999. http://www.ti3.tu-harburg.de/rump/.

[26] S. Theodorakis and C. Psaroudaki. Oscillations of a bose-einstein condensate in a rapidly contracting circular box. Physics Letters A, 373(4):441-447, 2009.

[27] W. Tucker. The Lorenz attractor exists. Comptes Rendus de l'Académie des Sciences-Series I-Mathematics, 328(12):1197-1202, 1999.

[28] W. Tucker. A rigorous ODE solver and Smale's 14th problem. Foundations of Computational Mathematics, 2(1):53-117, 2002.

[29] Jan Bouwe van den Berg and Jean-Philippe Lessard. Chaotic braided solutions via rigorous numerics: chaos in the Swift-Hohenberg equation. SIAM J. Appl. Dyn. Syst., 7(3):988-1031, 2008.

[30] Jan Bouwe van den Berg, Jason D. Mireles-James, Jean-Philippe Lessard, and Konstantin Mischaikow. Rigorous numerics for symmetric connecting orbits: even homoclinics of the Gray-Scott equation. SIAM J. Math. Anal., 43(4):1557-1594, 2011.

[31] Nobito Yamamoto. A numerical verification method for solutions of boundary value problems with local uniqueness by Banach's fixed-point theorem. SIAM J. Numer. Anal., 35(5):2004-2013 (electronic), 1998. 